\title{
Generation of cDC-like cells from human induced pluripotent stem cells via Notch signaling
}

\author{
Kenichi Makino, ${ }^{1,2}$ Mark D Long, ${ }^{3}$ Ryutaro Kajihara, ${ }^{1}$ Satoko Matsueda, ${ }^{1}$ \\ Takaaki Oba, ${ }^{1,4}$ Kazunori Kanehira, ${ }^{5}$ Song Liu, ${ }^{3}$ Fumito Ito (D) ${ }^{1,6,7,8}$
}

To cite: Makino K, Long MD, Kajihara R, et al. Generation of CDC-like cells from human induced pluripotent stem cells via Notch signaling. Journal for ImmunoTherapy of Cancer 2022;10:e003827. doi:10.1136/ jitc-2021-003827

- Additional supplemental material is published online only. To view, please visit the journal online (http://dx.doi.org/10. 1136/jitc-2021-003827).

KM, MDL and RK contributed equally.

Accepted 19 December 2021

Check for updates

(C) Author(s) (or their employer(s)) 2022. Re-use permitted under CC BY-NC. No commercial re-use. See rights and permissions. Published by BMJ.

For numbered affiliations see end of article.

Correspondence to

Dr Fumito Ito;

fumito.ito@med.usc.edu

\section{ABSTRACT}

Background Dendritic cells (DCs) play critical roles in regulating the innate and adaptive immune responses, and have long been a major focus of cancer immunotherapy. Accumulating evidence suggests that conventional type 1 DCs (cDC1s) excel in crosspresentation of exogenous antigens on MHC-I molecules and induction of antitumor $\mathrm{CD}^{+} \mathrm{T}$ cell immunity; however, obtaining large numbers of cDC1s is difficult. The use of reprogramming and differentiation technology is advantageous for obtaining unlimited numbers of autologous cDC1s especially for therapeutic interventions where repeated vaccinations are required. However, generation of cDC1s from human induced pluripotent stem cells (iPSCs) remains elusive.

Methods Human iPSCs established from peripheral blood T cells and monocytes were differentiated to myeloid cells under on-feeder or feeder-free culture conditions in vitro. Phenotype, genomic and transcriptomic signature, and function of human iPSC-derived DCs were analyzed. The role of Notch signaling for the generation of $\mathrm{HLA}-\mathrm{DR}^{+}$cells from human iPSCs was interrogated by a loss- and gainof-function approach.

Results Flow cytometric analyses and single-cell profiling of $\mathrm{HLA}-\mathrm{DR}^{+}$cells revealed that human iPSCs gave rise to $\mathrm{CD} 141^{+} \mathrm{XCR} 1^{+} \mathrm{CLEC9A^{+ }}$ cells

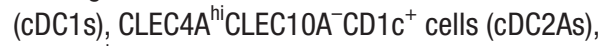
CLEC4A ${ }^{\text {lo }} \mathrm{CLEC}_{10 \mathrm{~A}^{+} \mathrm{CD}^{+} \mathrm{C}^{+} \text {cells (CDC2Bs), CD163 }}{ }^{-}$ $\mathrm{CD}^{+}{ }^{+} \mathrm{CD} 1 \mathrm{c}^{+}$cells $\left(\mathrm{CD}^{+}{ }^{+} \mathrm{CDC} 2 \mathrm{~s}\right)$, and $\mathrm{AXL}^{+} \mathrm{SIGLEC6}^{+}$cells (AS-DCs) on OP9 feeder cells expressing the Notch ligand delta-like 1 (OP9-DL1) while the majority of iPSC-derived cells differentiated on OP9 cells were $\mathrm{CD} 163^{+} \mathrm{CD} 5^{-} \mathrm{CD} 1 \mathrm{C}^{+}$ cells (DC3s) and monocytes. Plasmacytoid DCs were not differentiated from iPSCs on either OP9 or OP9-DL1 cells. Inhibition of Notch signaling during co-culture of iPSC-derived $\mathrm{CD} 34^{+}$hematopoietic progenitor cells with 0P9-DL1 cells abrogated generation of cDC1s, cDC2As, cDC2Bs, CD5 ${ }^{+}$CDC2s, and AS-DCs but increased frequency of DC3s. Notch-activated human iPSC-derived $\mathrm{XCR}^{+}{ }^{+} \mathrm{CLEC}_{\mathrm{A}}{ }^{+} \mathrm{HLA}-\mathrm{DR}{ }^{+} \mathrm{CD} 11 \mathrm{c}^{+}$cells exhibited similar gene expression profile with peripheral blood CDC1s. Human iPSC-derived DCs have phagocytic, T-cell proliferative, and cytokine-producing functions. Conclusions Our study demonstrates a critical role of Notch signaling in regulating developmental pathway of human cDCs. These findings provide insights into the future development of personalized treatment with unlimited numbers of autologous CDCs from human iPSCs.

\section{INTRODUCTION}

Dendritic cells (DCs) are a diverse population of specialized antigen-presenting cells that link innate and adaptive immunity, and crucial in the induction of immune responses to pathogens and tumors as well as for the maintenance of self-tolerance. ${ }^{1}$ DC-based immunotherapy has been tested for harnessing the potential of a patient's own immune system; however, which subsets of DCs can be used to design efficient vaccines remains to be determined. ${ }^{2}$ Although numerous clinical studies using monocytederived DC have showed DC vaccination has low toxicity and induces tumor-specific immune responses, the number of objective clinical responses is limited. ${ }^{2-4}$ Compelling evidence indicates that naturally occurring (primary) DC subsets are phenotypically and transcriptionally distinct from ex vivo-generated monocyte-derived DCs. ${ }^{3-11}$

Of the different naturally occurring DCs, the Batf3-dependent conventional type $1 \mathrm{DC}$ (cDC1) subset represents a promising alternative to monocyte-derived DCs for vaccination purposes. There is a growing body of evidence that cDC1s have the superior capacity to cross-present exogenous antigen to $\mathrm{CD}^{+} \mathrm{T}$ cells, produce IL-12 in response to innate and $\mathrm{T}$ cell-derived stimuli, induce Th1-type $\mathrm{T}$ cell responses, and play a critical role in antitumor T-cell responses. ${ }^{12-15}$ However, cDC1s are rare $(<0.2 \%-0.3 \%)$ in peripheral blood mononuclear cells (PBMCs), and isolating a sufficient number of cDC1s for repeated therapeutic vaccination is difficult. ${ }^{356}$

This limitation can be theoretically overcome by using induced pluripotent stem cells (iPSCs) as an unlimited source of autologous cDC1s. Previous studies reported successful generation of DCs from human iPSCs in co-culture with feeder cells in the presence of interleukin (IL)-4 and GM-CSF. ${ }^{16}{ }^{17}$ However, requirements for the generation of cDCls 
from human iPSCs remains to be clarified. Although previous studies showed differentiation of human iPSCs into CD $141^{+}$DCs without Notch signaling, ${ }^{18} 19$ emergent evidence revealed a key role of Notch signaling in differentiation of mouse and human hematopoietic CD $34^{+}$ progenitors into cDC1s. ${ }^{20} 21$

Here, we report efficient generation of functional cDC1-like cells from human iPSCs. Mechanistic studies illustrate non-redundant requirement of Notch signaling in differentiation of cDC1-like cells from human iPSCs. High-throughput characterization of human iPSCderived HLA-DR ${ }^{+}$cells not only shows the presence of DCs with cDC1 signatures resembling peripheral blood cDC1s but also reveals the heterogeneity of $\mathrm{CD}_{1} \mathrm{c}^{+} \mathrm{DCs}$ derived from human iPSCs.

\section{METHODS}

\section{Human samples}

After written informed consent, a venous blood sample of up to $30 \mathrm{~mL}$ was collected in EDTA tubes (BD Biosciences) from a healthy donor. PBMCs were isolated using Lymphocyte Separation Medium (Corning) density gradient centrifugation.

\section{Mice}

$\mathrm{NOD} / \mathrm{SCID} / \mathrm{IL}-2 \mathrm{R} \gamma$-deficient (NSG) mice were purchased from the Jackson Laboratories. Mice were 7 to 10 weeks old at the beginning of each experiment, and maintained under specific pathogen-free conditions and housed in the Laboratory Animal Resources facility. All animal studies were conducted in accordance with and approved by the Institutional Animal Care and Use Committee (IACUC) at the Roswell Park Comprehensive Cancer Center.

\section{Cell lines}

OP9 mouse bone marrow stromal cells and OP9 cells expressing the Notch ligand Delta-like-1 (OP9-DL1) were purchased from RIKEN (Japan). MS5-DL1 cells were purchased from MilliporeSigma. OP9-DL1 and MS5-DL1 cells express green fluorescent protein (GFP). OP9, OP9DL1, and MS5-DL1 cells were cultured on gelatin-coated dishes in OP9 medium: $\alpha$ MEM supplemented with 25\% non-heat inactivated fetal bovine serum (FBS; Biowest), $0.5 \%$ penicillin/streptomycin, and $2.2 \mathrm{~g} / \mathrm{L}$ of sodium bicarbonate (MilliporeSigma). SNL cells (Cell Biolabs, Inc.) were cultured in DMEM containing 7\% FBS (MilliporeSigma), $2 \mathrm{mM}$ L-glutamine, and $0.5 \%$ penicillin/ streptomycin. Human embryonic stem cells (ESCs) (H9; WiCell) were maintained on mitomycin C-inactivated SNL feeder cells in human ESC media which consisted of Primate ESC Medium (Reprocell) and $4 \mathrm{ng} / \mathrm{mL}$ basic fibroblast growth factor (bFGF; ThermoFisher Scientific), and used as controls for characterizing the pluripotency and differentiation capacity of generated iPSCs. These cell lines were authenticated by morphology, phenotype and growth, and routinely screened for Mycoplasma, and were maintained at $37^{\circ} \mathrm{C}$ in a humidified $5 \% \mathrm{CO}_{2}$ atmosphere.

\section{Establishment of iPSCs from peripheral blood T cells}

Peripheral blood $\mathrm{T}$ cells were reprogrammed to human iPSCs as described. ${ }^{22-24}$ In brief, $\mathrm{T}$ cells were activated with plate-bound anti-CD3 Ab $(10 \mu \mathrm{g} / \mathrm{mL})$ (BD Biosciences \#555336) and soluble anti-CD28 Ab $(5 \mu \mathrm{g} / \mathrm{mL})$ (BD Biosciences \#555725) in X-VIVO 15 medium (Lonza) containing 5\% FBS, 1 M HEPES (Gibco), $2 \mathrm{mM} \mathrm{L-glu-}$ tamine (Gibco), and penicillin/streptomycin (Gibco) and recombinant human IL-2 $(60 \mathrm{IU} / \mathrm{mL}$; Prometheus Laboratories Inc.) in a 24 -well plate at $1 \times 10^{6}$ cells/well for 5 days. On day 5 , T cells were reactivated with platebound anti-CD3 Ab and soluble anti-CD28 Ab and IL-2 $(60 \mathrm{IU} / \mathrm{mL})$ in a 24 -well plate at $1 \times 10^{5}$ cells/well for 24 hours. On day $6, \mathrm{~T}$ cells were infected with Sendai virus $(\mathrm{SeV})$ vectors that individually carried each of OCT3/4, SOX2, KLF4, and c-MYC (CytoTune-iPS 2.0 Reprogramming Kit; ThermoFisher Scientific) at 20 multiplicity of infection (MOI). After 24 hours of infection, the cells were collected and transferred to a $10 \mathrm{~cm}$ dish that contained mitomycin C-inactivated SNL feeder cells in human ESC media. The ESC medium was changed every other day until the colonies were picked. Then, the ESC culture medium was changed every day and the cells were passaged using $1 \mathrm{mg} / \mathrm{mL}$ collagenase Type IV (Invitrogen) every 5-6 days.

\section{Establishment and maintenance of iPSCs from peripheral blood monocytes}

Peripheral blood monocytes were reprogrammed to human iPSCs as described. ${ }^{25}{ }^{26}$ Briefly, the isolated PBMCs were incubated for 7 days in StemFit (AJINOMOTO, Tokyo, Japan) media containing cytokines, human IL-3 (20 ng/mL; Peprotech), IL-6 (50 ng/mL; Peprotech), thrombopoietin (TPO; $10 \mathrm{ng} / \mathrm{mL}$; Peprotech), stem cell factor (SCF; $50 \mathrm{ng} / \mathrm{mL}$; Peprotech), granulocyte colonystimulating factor (G-CSF; $10 \mathrm{ng} / \mathrm{mL}$; Peprotech, and FMS-like tyrosine kinase 3 ligand (Flt3L; $20 \mathrm{ng} / \mathrm{mL}$; R\&D Systems). The incubated monocytes were reprogrammed to iPSCs by transduction of reprogramming factors by $\mathrm{SeV}$ using CytoTune-iPS 2.0 Reprogramming Kit at 20 MOI. The reprogrammed cells were cultured on iMatrix-511 or iMatrix-511 silk (Nippi, Tokyo, Japan) coated plate and their medium was replaced with StemFit iPSC medium containing bFGF. The established iPSCs were cloned and their pluripotency characterized by expression of markers and teratoma formation in immunodeficient mice.

\section{Gene expression analysis by RT-PCR}

Total RNA samples were isolated with TRIzol reagent (Life Technologies) and purified, according to the manufacturer's instructions. One microgram total RNA was used for cDNA synthesis by RT-PCR with SuperScript III Reverse Transcriptase (Life Technologies) and random primer, according to the manufacturer's instructions. cDNA was amplified by PCR with Platinum Taq DNA Polymerase 
(Life Technologies) and various sets of primers showed in online supplemental table S1.

\section{Immunocytochemistry}

Human iPSCs were cultured on iMatrix-511 coated plate and incubated for 5 to 7 days. After formation of iPSC colonies, they were fixed with $4 \%$ paraformaldehyde. Immunofluorescence staining was performed using the following primary antibodies: anti-OCT3/4 antibody (Santa Cruz Biotechnology); anti-SSEA4 antibody (MilliporeSigma). Alexa Fluor 488-conjugated anti-mouse IgG antibody (Jackson ImmunoResearch) was used for the secondary antibody.

\section{Generation of DCs from human iPSCs on feeder cells}

To generate hematopoietic progenitor cells (HPCs), iPSCs were co-cultured with OP9 cells as described. ${ }^{17}$ In brief, human iPSCs $\left(2 \times 10^{4} /\right.$ well $)$ were cultured in a 6-well plate for 7 days before differentiation. Human iPSCs from two wells of the 6 -well plate were gently detached by a cell scraper, and placed on a gelatin pre-coated OP9 overconfluent $10 \mathrm{~cm}$ dish in $10 \mathrm{~mL}$ of $\alpha$ MEM containing $10 \%$ FBS and monothioglycerol $(100 \mu \mathrm{M}$; MilliporeSigma) (day 0 ). Medium was replaced by $20 \mathrm{~mL}$ of fresh medium on day 1 , and half of the volume of them was replaced on days $4,6,8$, and 11 . On day 13 , colonies were treated for $30 \mathrm{~min}$ with $5 \mathrm{~mL}$ of collagenase Type IV $(50 \mathrm{U} / \mathrm{mL})$ and subsequently dissociated for $30 \mathrm{~min}$ at $37^{\circ} \mathrm{C}$ using $5 \mathrm{~mL}$ of $0.05 \%$ Trypsin-EDTA (Gibco). To remove stromal cells, dissociated cells were resuspended adding 5 times OP9 medium plated on plastic at $37^{\circ} \mathrm{C}$ for $45 \mathrm{~min}$ and floating cells were collected. To further remove stromal cells and aggregated cells, cells were passed through a $70 \mu \mathrm{m}$ filter. $\mathrm{CD} 34^{+}$cells were isolated from the generated cell population by MACS (Magnetic activated cell sorting; Miltenyi Biotec), and placed on gelatin pre-coated OP9 or OP9DL1 cells in $10 \mathrm{~mL}$ of $\alpha \mathrm{MEM}$ containing $10 \%$ FBS with GM-CSF (20 ng/mL; Peprotech), Flt3L (100 ng/mL), and SCF $(20 \mathrm{ng} / \mathrm{mL})$ for 2 weeks. Medium was replaced once a week. In some experiments, we added a $\gamma$-secretase inhibitor, N-[N-(3,5-difluorophenacetyl)- L-alanyl]-Sphenylglycine t-butyl ester (DAPT; $5 \mu \mathrm{M}$; MilliporeSigma \#565790) or DMSO when we co-culture human iPSCderived CD $34^{+}$cells with OP9-DL-1 cells.

\section{Generation of DCs from human iPSCs on feeder-free condition}

We added Flt3L to the previously described protocol ${ }^{19}$ for generation of CD141 ${ }^{+} \mathrm{XCR} 1^{+} \mathrm{CLEC} 9 \mathrm{~A}^{+} \mathrm{HLA}-\mathrm{DR}^{+}$cells under feeder-free condition. In brief, we cultured human iPSCs in $4 \mathrm{~mL} /$ well of XVIVO-15 in a 6-well plate supplemented with $1 \mathrm{mM}$ sodium pyruvate (Gibco), $0.1 \mathrm{mM}$ non-essential amino acid (NEAA, Gibco), $2 \mathrm{mM}$ L-glutamine, and $0.05 \mathrm{mM}$ 2-mercaptoethanol (Gibco) in the presence of the cytokine cocktail containing Flt3L $(1 \mu \mathrm{g} /$ $\mathrm{mL})$, GM-CSF $(50 \mathrm{ng} / \mathrm{mL})$, SCF $(20 \mathrm{ng} / \mathrm{mL})$, vascular endothelial growth factor (VEGF, $50 \mathrm{ng} / \mathrm{mL}$; Peprotech), and bone morphogenetic protein 4 (BMP4; $50 \mathrm{ng} / \mathrm{mL}$; Peprotech). Half of the medium was replaced every 2 days from day 2 of culture. BMP4 was removed from day 5 onward, followed by VEGF (day 14) and SCF (day 19). On days 14-28 of culture, IL-4 (Peprotech) was added to the medium at $10 \mathrm{ng} / \mathrm{mL}$ (days 14,15 ), $20 \mathrm{ng} / \mathrm{mL}$ (days $16,17), 40 \mathrm{ng} / \mathrm{mL}$ (days 18, 19), $60 \mathrm{ng} / \mathrm{mL}$ (days 20, 21), $80 \mathrm{ng} / \mathrm{mL}$ (days 22, 23), and $100 \mathrm{ng} / \mathrm{mL}$ (days 24-28). DCs were harvested on day 28 using gentle pipetting.

\section{Teratoma formation and histological analysis}

Undifferentiated human iPS cells $\left(1 \times 10^{6}\right)$ suspended in $100 \mu \mathrm{L}$ of DMEM containing $10 \%$ FBS were injected into the subcutaneous tissue of NSG mice. Four weeks after injection, tumors were surgically dissected from the mice. Samples were fixed in PBS containing 10\% paraformaldehyde and embedded in paraffin. Paraffin-embedded samples were stained with H\&E for histological analysis.

\section{Cytology}

Human iPSC-derived differentiated cells were harvested and Hema 3 leukocyte staining were performed. Briefly, $5 \times 10$ cells in $100 \mu \mathrm{L}$ PBS were centrifuged on microscope slides using a Shandon Cytospin 2 Cytocentrifuge (Thermo Electron). The cytocentrifuged cell smears were fixed and stained with Hema 3 stain kit (Fisher Scientific).

\section{Microscopy}

Images of Hema 3-stained cytospins and H\&E-stained teratoma were taken on an Olympus BX45TF inverted microscope with an Olympus DP27 digital camera.

\section{Flow cytometry}

Phenotypic analysis of PBMCs and human iPSC-derived cells was performed as described. ${ }^{22}{ }^{24} 27$ Briefly, single cell suspensions of PBMCs and human iPSC-derived cells were stained with a master mix of antibodies (Abs) for surface stains after Fc block (human Fc block; BD Biosciences) for $20 \mathrm{~min}$ at room temperature. Antibodies used in this study are listed in online supplemental table S2. Live/ Dead Fixable Aqua Dead Cell Stain Kit (ThermoFisher Scientific) and LIVE/DEAD Fixable Near-IR Dead Cell Stain kit (ThermoFisher Scientific) were used to exclude dead cells from the analysis. Samples were analyzed using LSRII or LSRFortessa (BD Biosciences) with FlowJo software (TreeStar).

\section{Functional characterization of generated human iPSC-DCs}

Cell surface phenotype was analyzed by flow cytometer. Phagocytosis was evaluated using the pHrodo Green Zymosan Bioparticles (ThermoFisher Scientific), as described. ${ }^{18}$ In proliferation assay, peripheral blood $\mathrm{T}$ cells were isolated by immunomagnetic negative selection from the same blood donor of iPSCs using EasySep Human $\mathrm{T}$ cell Isolation Kit (STEMCELL Technologies). T cells were labeled by Carboxyfluorescein Succinimidyl Ester (CellTrace CFSE Cell Proliferation Kit; ThermoFisher Scientific) and cultured with HLA-DR ${ }^{+}$iPSC-DCs at $37^{\circ} \mathrm{C}$ with $5 \% \mathrm{CO}_{2}$ in presence of positive control peptide pool of major histocompatibility complex (MHC) class I-restricted and II-restricted epitopes (CEFX-3 and CEFX-4; 
JPT Peptide Technologies) and IL-2 (60IU/mL). In some experiments, we magnetically sorted CLEC9A ${ }^{+}$ cells by staining with PE anti-human CLEC9A followed by anti-PE MicroBeads (Miltenyi Biotec) before co-culture with CFSE-labeled T cells. The DC:T cell ratio was $1: 5$. At day 5 , cells were harvested and analyzed by flow cytometer. To identify cytokine production from human iPSC-derived DCs, cells were stimulated with an activation cocktail consisting of polyinosinic:polycytidylic acid (poly(I:C), $25 \mu \mathrm{g} / \mathrm{mL}$; InvivoGen \#tlrl-pic), R848 (2.5 $\mu \mathrm{g}$ / mL; InvivoGen \#tlrl-r848), CpG oligodeoxynucleotides (ODN) 2216 ( $1 \mu \mathrm{M}$; InvivoGen \#tlrl-2216), and lipopolysaccharide (LPS, $100 \mathrm{ng} / \mathrm{mL}$; MilliporeSigma \#L6529) for 6 hours at $37^{\circ} \mathrm{C}, 5 \% \mathrm{CO}_{2}$. BD GolgiPlug (BD Biosciences) was added to the culture in last 4 hours.

\section{Single cell RNA sequencing (scRNAseq)}

\section{Sample preparation}

For the scRNAseq experiments, single cell suspensions of human iPSC-derived DCs were stained with a master mix of antibodies for surface staining including Live/Dead Fixable Aqua Dead, CD11c, and HLA-DR after Fc block. Sorting of live HLA-DR ${ }^{+}$cells were performed on a BD FACSAria II (BD Biosciences).

\section{Single-cell RNA sequencing library generation}

Droplet-based 3' end massively parallel scRNAseq was performed by encapsulating flow-cytometry-sorted cells into droplets and libraries were prepared using Chromium Single Cell 3' Reagent Kit V3 according to manufacturer's protocol (10x Genomics). To minimize batch effect, library preparation was performed on all captured cells simultaneously, and all libraries were sequenced on a single Novaseq S1 flow cell on an Illumina Novaseq 6000 instrument.

Raw data processing, quality control for cell inclusion, and scRNAseq analysis

Raw sequence data demultiplexing, barcode processing, alignment (mm10), and filtering for true cells were performed using the Cell Ranger Single-Cell Software Suite (V.5.0.0), yielding cells (OP9: 5753 cells, and OP9DL1: 4158 cells) with a mean of 29,643 reads / cell $(97.60 \%$ mapping rate), median of 3172 genes/cell, 20,142 total unique detectable genes, and 11,353 median UMI counts/cell (online supplemental table S3). Subsequent filtering and downstream analyses were performed using Seurat (v3) ${ }^{28}$ Genes expressed in less than 3 cells and cells that express less than 350 genes were excluded from further analyses. Additional filtering of cells was determined based on the overall distributions of total RNA counts $(<55,000)$ and the proportion of mitochondrial genes $(<12 \%)$ detected to eliminate potential doublets and dying cells, respectively. Additional detection of doublets was performed using Scrublet. ${ }^{29}$ Thresholding for doublet detection was set based on total distribution of doublet scores (doublet threshold=0.2). Quantification of mitochondrial and ribosomal gene expression was calculated using the PercentageFeatureSet function, using gene sets compiled from the Mouse Genome Informatics database. Cell cycle phase scoring was accomplished against normalized expression via the CellCycleScoring function using mouse genes orthologous to known cell cycle phase marker genes. ${ }^{30}$ Ultimately, we removed 982 cells $(9.91 \%$ of total cells) after quality control assessment and included 8929 cells (OP9: 5283 cells and OP9-DL1: 3646 cells) for analysis. Normalization and variance stabilization were conducted using regularized negative binomial regression (sctransform) and sample data subsequently harmonized using anchor-based integration implemented with Seurat v3. Principle component analysis (PCA) was performed on integrated data and optimal dimensionality of the dataset was decided by examination of the Elbow plot as the total number of PCs where gain in cumulative variation explained was greater than $0.1 \% \quad(\mathrm{PCs}=40)$. The FindNeighbors function was used that implements a graph-based nearest neighbor clustering approach, and then the FindClusters function was used to identify final cell clusters $(n=13)$ using a resolution of 0.08 . Uniform manifold approximation and projection (UMAP) was applied for non-linear dimensional reduction to obtain a low dimensional representation of cellular states. Differential expression between clusters was determined using the MAST method via the FindMarkers function, using a minimum expression proportion of $25 \%$ and a minimum log fold change of 0.25 . Unbiased cell type annotation was performed using SingleR. ${ }^{31}$ Briefly, this framework allows for the annotation of scRNAseq data to reference transcriptome data sets of known origin to infer the cellular state of each input cell. The built in Novershtern Hematopoietic reference $^{32}$ was used for SingleR-based cell annotation. Mean expression of markers found within each cluster or cell annotation were used for subsequent analyses including heatmap visualization and pathway analysis. Referencebased mapping of hiPSC-DC-derived scRNA-seq data was performed against previously published datasets characterizing DC heterogeneity in human PB and spleen. ${ }^{33-36}$ Raw counts were obtained from the Gene Expression Omnibus (GSE137710, GSE94820, GSE151087, GSE132566) and re-processed using a Seurat-based pipeline described previously. Cell annotations were obtained as reported in initial publication (GSE137710, GSE94820) or inferred from custom analysis using manuscript specific gene markers (GSE132566, GSE151087). Gene sets for single-cell enrichment analyses were pulled directly from reported cell type gene markers from each respective study. Reference-based mapping and label transfer of previously annotated cells was performed using the FindTransferAnchors and MapQuery functions implemented within Seurat. Reference-based mapping of hiPSC-DC-derived scRNA-seq data to the Azimuth PBMC global reference was performed using Azimuth (https:// azimuth.hubmapconsortium.org/). 


\section{Pathway analysis}

Gene set enrichment analysis (GSEA) of select differential expression profiles identified between groups or clusters was done using enrichR and clusterProfiler in R. Single-cell functional enrichment analysis was done using AUCell, ${ }^{37}$ which applies an area under the curve method to query cell-to-cell pathway activity which is robust to noise typical of scRNAseq datasets. Six pathway databases (Hallmark, KEGG, Reactome, PID, BioCarta, and GO-BP) were compiled from the Molecular Signatures Database (MSigDB) ${ }^{38}$ and used as a reference sets for functional enrichments. For GSEA, only gene sets with fdr adjusted $\mathrm{p}<0.05$ were considered as significantly enriched. To visualize select functional enrichments, we generated heatmaps and/or lollipop plots of normalized enrichment scores (NES) of relevant biological pathways.

\section{RNA Sequencing}

The generated iPSC-derived DCs and PBMCs were stained with a master mix of antibodies for surface staining including Live/Dead Fixable Aqua Dead, XCR1, CLEC9A, CD11c, and HLA-DR after Fc block with human IgG. CD11 ${ }^{+}$HLA-DR ${ }^{+}$CLEC9A ${ }^{+} \mathrm{XCR}^{+}{ }^{+}$cells were sorted on BD FACSAria II. Total RNA was prepared from these sorted DCs using the miRNeasy mini kit (Qiagen). TruSeq Stranded messenger RNA (mRNA) (Illumina) was used for library preparation. Quality control for raw reads was performed with fastqc. Transcript abundances were quantified from sequencing reads against the human reference genome (GRCh37.67) using salmon-0.14.1. ${ }^{39}$ Raw feature counts were normalized and differential expression analysis carried out using DESeq2. ${ }^{40}$ Differential expression rank order was used for subsequent GSEA, performed using the clusterProfiler package in R. Gene sets queried included the Hallmark, Canonical pathways, and GO Biological Processes Ontology collections available through the Molecular Signatures Database (MSigDB) ${ }^{38}$

\section{RESULTS \\ Notch signaling facilitates generation of ${\text { CD } 141^{+} \text {CLEC9A }}^{+}$XCR1 ${ }^{+}$DCs from human iPSCs}

Recent work revealed Notch-dependent differentiation of $\mathrm{Lin}^{-} \mathrm{CD} 34^{+} \mathrm{CD} 43^{+} \mathrm{CD} 45^{+}$myeloid progenitors into $\mathrm{cDC}_{1},{ }^{20}{ }^{21}$ while previous studies showed differentiation of human iPSCs into CD141 ${ }^{+}$DCs without Notch signaling. ${ }^{18} 19$ Therefore, it remains unclear whether Notch signaling is required for the generation of cDC1s from human iPSCs. To this end, we first sought to establish iPSCs from peripheral blood $\mathrm{T}$ cells using the $\mathrm{SeV}$ vector reprogramming system which has been shown to efficiently reprogram human and murine $\mathrm{T}$ cells. ${ }^{2341}$ Peripheral blood T cells were transduced with reprogramming factors via $\mathrm{SeV}$ vectors 24 hours after activation with anti-CD3 and anti-CD28 Ab. ${ }^{22-24}$ Twenty-four hours after gene introduction, the cells were replated onto feeder layers of SNL cells with ESC medium containing bFGF. To compare the differentiation capacity of human
iPSCs of different origin and established by different methods, we also generated iPSCs from peripheral blood monocytes. PBMCs from the same donor were cultured with mononuclear cell medium containing IL-6, SCF, TPO, Flt3L, IL-3, and G-CSF for 7 days to enrich monocytes. ${ }^{2526}$ Peripheral blood monocytes were transduced by the $\mathrm{SeV}$ reprogramming system and, 24 hours later, transferred onto laminin-coated plate in StemFit medium containing bFGF. The established iPSCs derived from $\mathrm{T}$ cells (TiPSCs) and monocytes (MiPSCs) had morphology typical of human ESCs (online supplemental figure 1A). RT-PCR analyses showed that iPSCs expressed transcripts for the ESC marker NANOG, whereas they have lost $\mathrm{SeV}$ transcripts early on after a few passages (online supplemental figure 1B). Immunostaining revealed that iPSCs expressed the OCT3/4 and SSEA4 proteins (online supplemental figure 1C). On subcutaneous injection into NSG mice, human iPSCs gave rise to teratomas representing all three embryonic germ layers (online supplemental figure 1D).

For hematopoietic differentiation of human iPSCs, we used the bone marrow stromal cell line, OP9, as a feeder as previously described. ${ }^{17}$ We cultured human iPSCs on OP9 cells for 13 days and observed mesodermal colonies (online supplemental figure 2A). To analyze phenotype of differentiated cells, cells were gated on the pan-human marker CD147 to gate out the fluorescent signal from remaining murine OP9 cells (online supplemental figure 2B). We found the majority of human ESC-derived and iPSC-derived CD $34^{+}$cells expressed CD43 and/or CD45 (online supplemental figure 2C). To determine the role of Notch signaling in generation of cDC1s, we isolated $\mathrm{CD}_{4} 4^{+}$cells from day 13 of co-culture and cultured them on OP9 or OP9-DL1 cells in the presence of SCF, GM-CSF, and Flt3L for 14 days (figure 1A). Approximately $1 \times 10^{6}$ cells were differentiated from $0.5 \sim 2 \times 10^{5}$ iPSCs on OP9 or OP9-DL1 cells. The analysis of cell morphology via cytospins revealed that differentiated cells were found to be myelomonocytic cells; mixture of granulocytic and monocytic cells was identified on OP9 feeder cells while myeloid cells with dendritic projections and veils typical of cells of dendritic lineage found on OP9-DL1 feeder cells (figure 1B).

We next aimed to define TiPSC-derived cells by conventional flow cytometry (online supplemental figure 3A). We found that co-culture with OP9-DL1 cells yielded substantially more HLA-DR ${ }^{+}$cells compared with control OP9 cells which had more monocytic cells expressing CD14, CD16, and/or CD163 (figure 1C). The majority of HLA-DR ${ }^{+}$ cells differentiated on OP9 cells were $\mathrm{CD} 1 \mathrm{c}^{+} \mathrm{CD} 141^{-} \mathrm{DCs}$. In contrast, DL1-Notch signaling markedly increased the frequency of $\mathrm{CD} 141^{+} \mathrm{CLEC} 9 \mathrm{~A}^{+} \mathrm{XCR} 1^{+}$DCs (figure 1D). These cells also expressed CD1c consistent with previous studies showing CD1c expression in $\mathrm{CD} 141^{+} \mathrm{cDC} 1 \mathrm{~s}$ differentiated from human $\mathrm{CD} 34^{+}$cord blood progenitors in vitro and in Flt3L-induced CD141 ${ }^{+}$cDC1s in vivo. ${ }^{20} 4243$ We found higher frequency of HLA-DR ${ }^{+}$cells expressing CD80, CD40, and CCR7 in coculture with OP9-DL1 
A

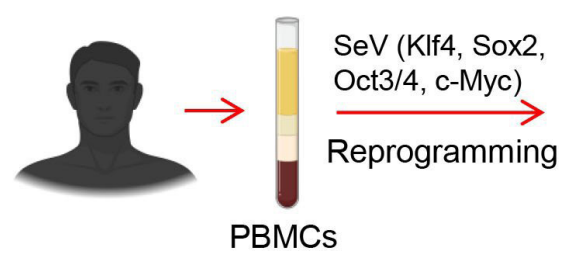

Suppl. figure 1

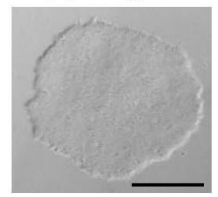

Human iPSCs
Suppl. figure 2

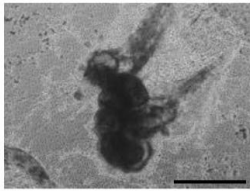

Isolate CD $34^{+}$cells
GM-CSF,

Flt3L, SCF Harvest nonadherent cells

B

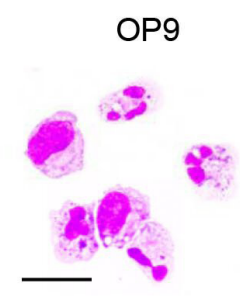

OP9-DL1

C

E
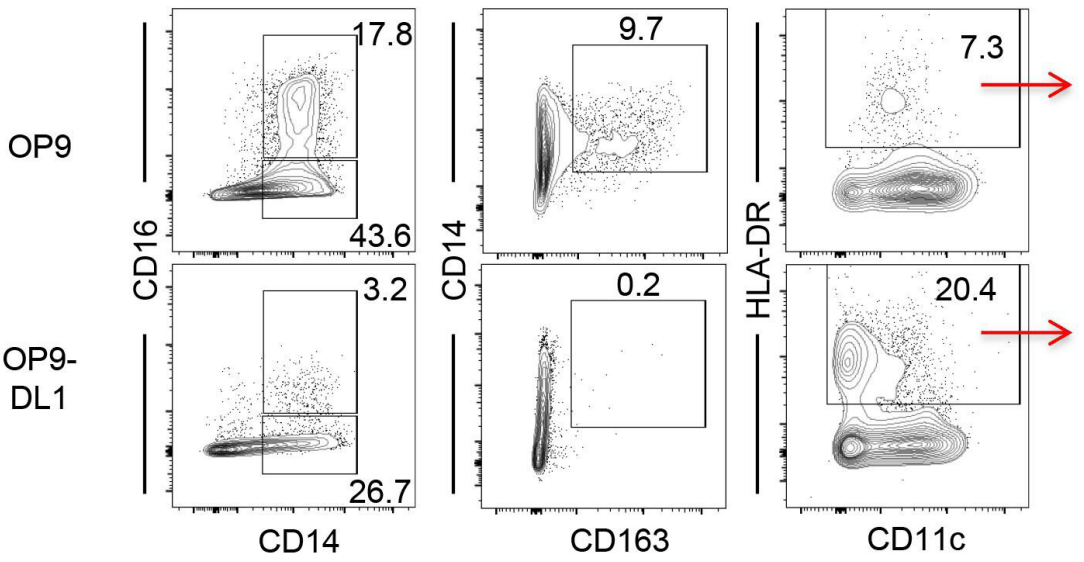

D
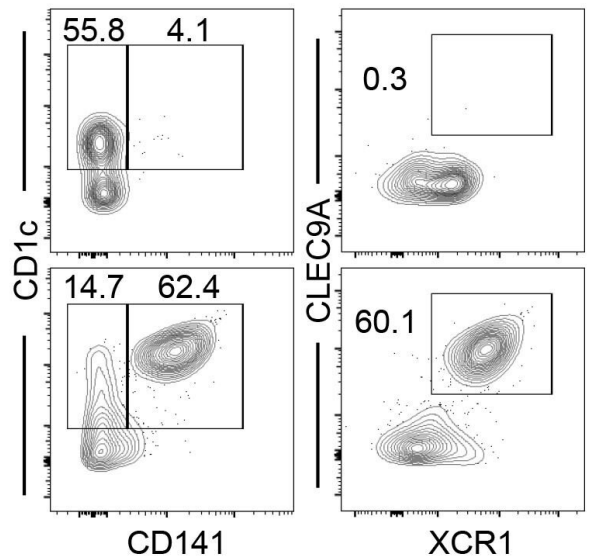

(Gated on HLA-DR ${ }^{+}$cells)

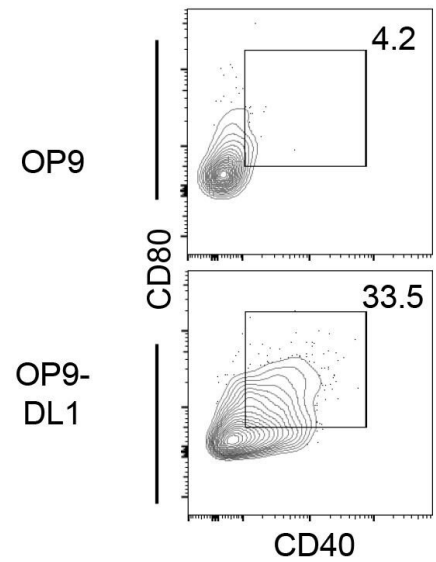

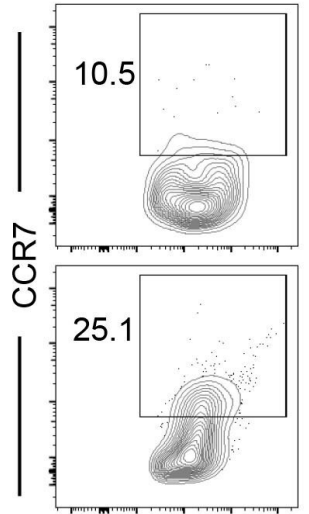

HLA-DR

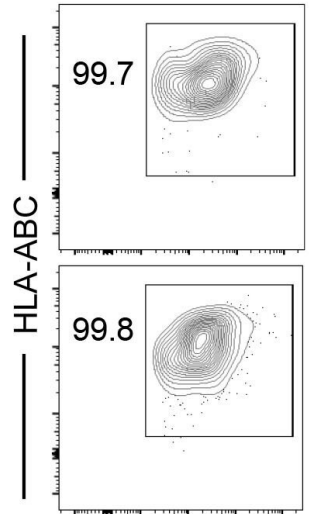

HLA-DR

Figure 1 Notch signaling facilitates generation of CD $141^{+} \mathrm{CLEC} 9 \mathrm{~A}^{+} \mathrm{XCR} 1^{+} \mathrm{DCs}$ from human iPSCs. (A) Schematic illustration showing the generation of DCs from iPSCs (scale bar, $500 \mu \mathrm{m})$. (B) Hema 3-stained cytospins of human iPSC-derived cells on OP9 (left) and OP9-DL1 (right) feeder cells (scale bar, 10 $\mathrm{mm}$ ). (C-E) Representative flow cytometric plots showing marker expression of human iPSC-derived cells on OP9 (upper) and OP9-DL1 (lower) feeder cells. Number denotes percent positive cells for each marker. Data shown are representative of three independent experiments.

compared with OP9 cells although there was no difference in HLA-ABC expression (figure 1E). Human ESCs and another TiPSC-derived clone (\#2) similarly differentiated to cDC1-like cells on OP9-DL1 but not on OP9 cells (online supplemental figure 3B,C). We also examined whether TiPSCs could differentiate to cDC1-like cells on different feeder cells, MS5 expressing Notch ligand DL1 (MS5-hDLL1), and found that Notch signaling facilitated generation of $\mathrm{CD} 141^{+} \mathrm{CLEC} 9 \mathrm{~A}^{+} \mathrm{XCR} 1^{+}$DCs regardless of the type of feeder cells (online supplemental figure 3D). Furthermore, we addressed whether there was phenotypical difference between TiPSCs and MiPSCs differentiated 
on OP9-DL1 cells. MiPSCs also gave rise to cDC1-like cells expressing CD141 ${ }^{+}$and CLEC9A ${ }^{+}$(online supplemental figure 4A). We found both TiPSC-derived and MiPSCderived HLA-DR ${ }^{+}$cells contained comparable frequency of $\mathrm{CD} 80^{+}, \mathrm{CD} 40^{+}$, and $\mathrm{TLR}^{+}$cells (online supplemental figure 4B). Hereafter, TiPSC-derived DCs were used for further analyses.

\section{Identification of various human iPSC-derived CDC subsets by single-cell RNA sequencing}

Next, we carried out single-cell RNA-sequencing (scRNAseq) to further characterize iPSC-derived HLA$\mathrm{DR}^{+}$cells. The corresponding fluorescence-activated cell sorting (FACS) - purified single live $\mathrm{CD} 147^{+} \mathrm{GFP}^{-} \mathrm{HLA}-\mathrm{DR}^{+}$ cell populations differentiated on OP9 and OP9-DL1 cells were subjected to scRNAseq by using the 10x Genomics pipeline. This yielded data for 8929 high-quality cells after stringent filtering (OP9: 5283 cells; OP9-DL1: 3646 cells) (online supplemental figure 5A,B). Unsupervised clustering analysis identified 12 myelomonocytic clusters (cluster (C) $0-10,12)$ and a small cluster of erythroids (C11) (figure 2A-C and online supplemental figure 6). Human iPSCs differentiated on OP9-DL1 contained markedly increased frequency of DCs (C3, 5, 6, and 8) characterized by high levels of MHC class II-related genes (HLA-DQB1, HLA-DQA1, HLA-DRB1, HLA-DRB5, HLA-DPA1, and HLA-DPB1) (figure 2B-D and online supplemental figure 7). In contrast, a majority of OP9differentiated iPSC-derived cells were computationally identified as monocytes (C0-2, 7, 9, 10, 12) and colony forming unit-monocytes (CFU-M: C4) (figure 2A-E and online supplemental table S4). Within the clusters expressing higher levels of MHC class II genes, C6 expressed genes and transcriptomes specific to cDC1s $^{1120}$ such as DNASE1L3, CADM1, SLAMF8, SNX22, GCSAM, CLNK, CLEC9A, BATF3, and XCR1, and antigen processing and presentation pathway, and almost exclusively appeared on OP9-DL1 feeder cells (figure 2C-F and online supplemental figures $\mathrm{S} 6,7$ ).

Recent studies employing scRNAseq have identified new human DC populations and elucidated the heterogeneity of CD1c ${ }^{+}$DCs. ${ }^{33-3644-46}$ In our study, C3, 5, and 8 expressed $C D 1 C$, and $\mathrm{C} 3$ was notable for increased expression of cell cycle-associated genes (MKI67, STMN1, $C C N B 1$, and KPNA2) (figure 2C and online supplemental figure S7) and pathway (figure $2 \mathrm{~F}$ ), consistent with mitotic DCs. ${ }^{33}$ Expression of CLEC1OA, the hallmark peripheral blood cDC2 gene, was low in C8; however, C8 exhibited higher levels of CLEC4A, RUNX3, LTB, IL22RA2, and IDO1 resembling the cDC2A subset, recently identified in human spleen (figure 2D and online supplemental figure S7) ${ }^{33}$ This subset was found mainly on OP9-DL1 feeder cells, and was notable for high expression of $C D 3 E$, which can be induced by Notch signaling. ${ }^{47}$ C5 expressed higher levels of the canonical cDC2 markers, CLEC1OA and FCER1A, suggesting cDC2Bs. ${ }^{33}$

To confirm the generation of cDC2A from iPSC-derived cells, we used scRNAseq data derived from a recent study by
Brown et al evaluating Lin(CD3, CD56, CD19) ${ }^{-} \mathrm{CD} 14^{-} \mathrm{CD}-$ $11 \mathrm{c}^{+} \mathrm{HLA}-\mathrm{DR}^{+}$cells isolated from human spleen. ${ }^{33}$ Using an integration-based approach, we projected DC subsets, C3, 5, 6, and 8, in our scRNAseq data onto the previously annotated cells reported by Brown $e t a l^{33}$ to generate a joint UMAP representation and infer DC cell states (figure 3 and online supplemental figure S8A). We found that iPSC-derived cells generated on OP9-DL1 feeder cells contained cDC1s and cDC2Bs and also cDC2As (figure 3B and online supplemental figure S8B). Consistent with these results, flow cytometric analysis of iPSCderived $\mathrm{CD}^{-} 6^{-} \mathrm{CD} 88^{-} \mathrm{CD} 141^{-} \mathrm{HLA}^{-D R^{+}}$cells identified CLEC4 $\mathrm{A}^{\text {hi }}$ CLEC10A ${ }^{-} \mathrm{CD} 1 \mathrm{c}^{+}$cells representing cDC2A as

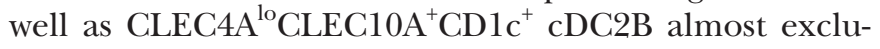
sively on OP9-DL1 feeder cells (figure 3C).

A previous report has shown that peripheral blood cDC2s contained $\mathrm{CD}^{+}$and $\mathrm{CD}^{-}$subsets with distinct gene expression and functions. ${ }^{48}$ Also, high-dimensional single-cell analysis of PBMCs identified new DC subsets expressing AXL and SIGLEC6, named "AS-DCs". ${ }^{36} 44$ In our study, $\mathrm{CD} 163^{-} \mathrm{CD}^{+} \mathrm{CD} 1 \mathrm{c}^{+} \mathrm{DCs}\left(\mathrm{CD}^{+} \mathrm{CDC} 2 \mathrm{~s}\right)$ and AS-DCs were only identified in iPSC-DCs on OP9-DL1 feeder cells on flow cytometric analysis (figure 3C and $\mathrm{D}$ and online supplemental figure 9). Consistent with this, we observed cells expressing AXL, SIGLEC6, and/ or $C D 5$ within the DC clusters, C3, 5, 6, and/or 8 in our scRNAseq data (online supplemental figure 10A,B). Of note, there was an overlap between $C D 5$-expressing cells and AS-DCs in our and Brown et al's scRNAseq data (online supplemental figure 10C,D) in agreement with previous studies. ${ }^{36446}$

$\mathrm{CD}_{1}{ }^{+}$DCs expressing classical cDC2-related and monocyte-related genes have been identified, and named "DC3s". ${ }^{36}$ DC3s are phenotypically defined as CD88$\mathrm{CD} 16^{-} \mathrm{CD} 163^{+} \mathrm{CD}^{-} \mathrm{CD}^{-} \mathrm{c}^{+}$with low to high expression of CD14. ${ }^{343545} 46$ Flow cytometric analysis revealed that the majority of iPSC-derived DC3s were found on OP9 feeder cells unlike cDC1s, cDC2As, cDC2Bs, $\mathrm{CD} 5^{+} \mathrm{cDC} 2 \mathrm{~s}$, and AS-DCs differentiated on OP9-DL1 cells (figure 3C). This is consistent with distribution of cells expressing $\mathrm{CD} 163$, CD36, CD14, and F13A1 (online supplemental figure 11 ), which are highly positive on DC3 ${ }^{36}$ However, there were few cells co-expressing CDKN1A and LMNA, genes which are selectively expressed to higher levels in DC3s, ${ }^{35}$ and some cells may also have expressed C3AR1 and/or C5AR1 encoding CD88, which are positive on monocytes, monocyte-derived DCs, and macrophages but negative on cDC2s and DC3s. ${ }^{34-36}$ To clarify this issue, we projected our scRNAseq data onto the previously annotated cells reported by Villani et al, Bourdely et al, and Dutertre et al to confirm the presence of iPSC-derived DC3s. Using a reference map and cell annotations derived from Villani et al (online supplemental figure 12A), we found that a distinct subset of DC3s could be identified when projecting total cells (online supplemental figure 12B) but not DC clusters C3, 5, 6, and 8 (online supplemental figure 12C). Single-cell enrichment scores using the gene set specific to DC3 cells determined by Villani et al were 
A
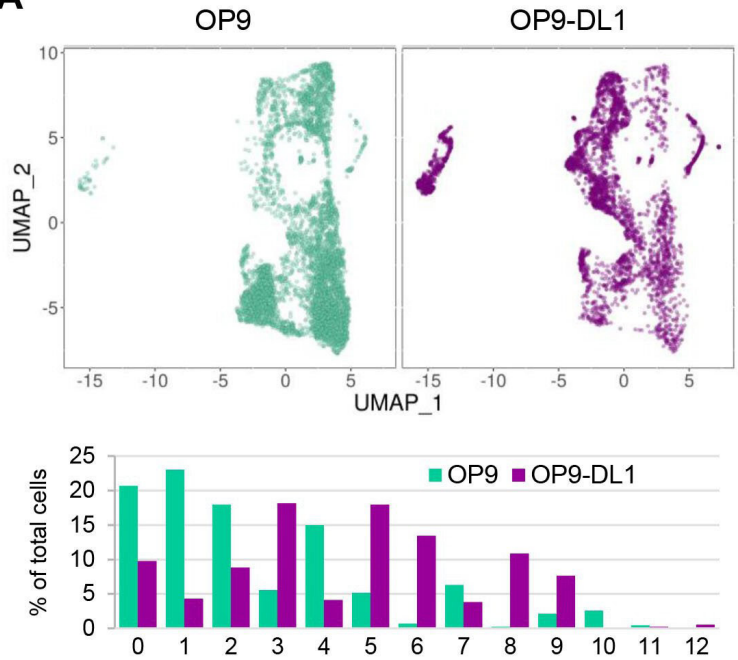

D

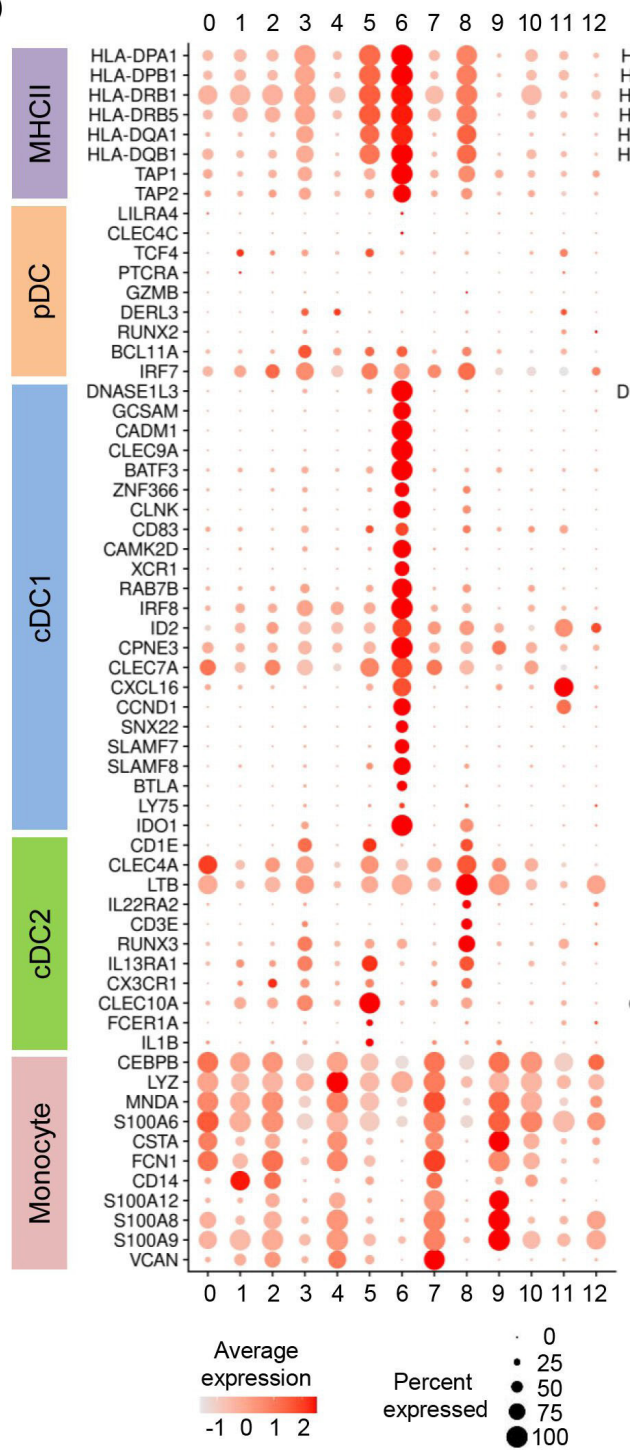

B

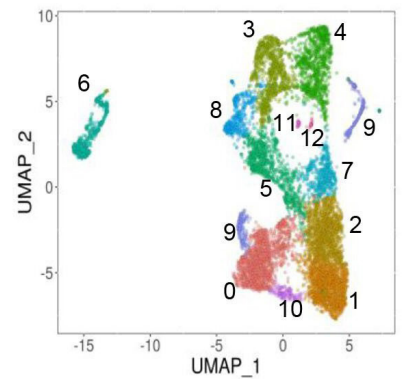

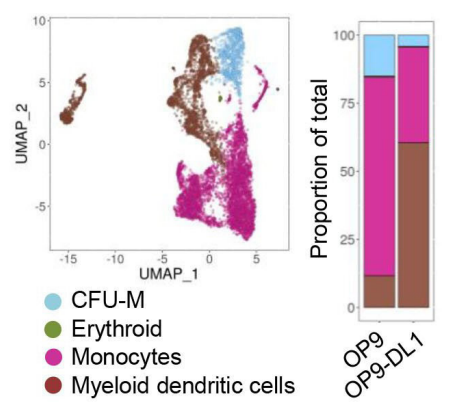

C

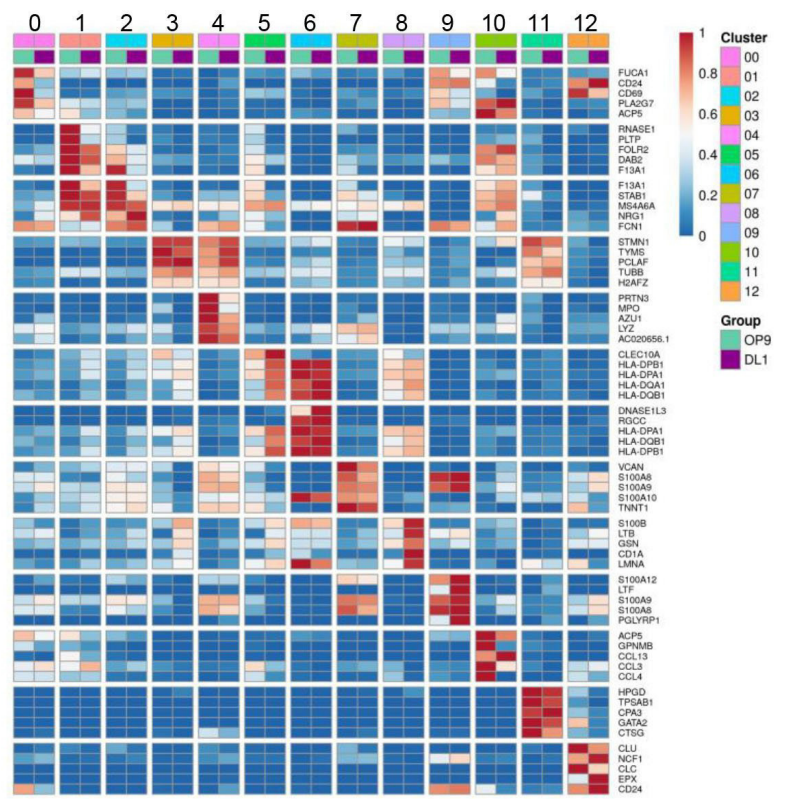

E

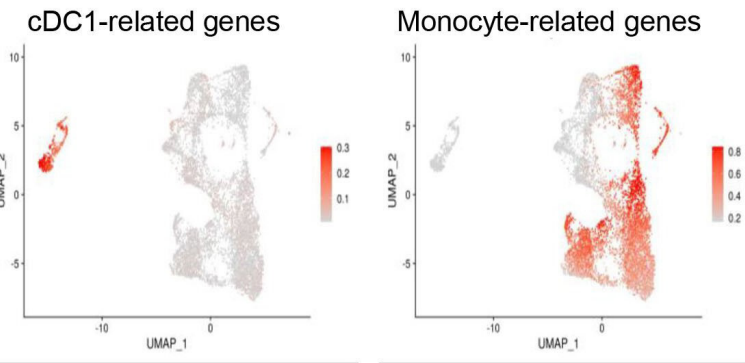

F KEGG_antigen_processing _and_presentation

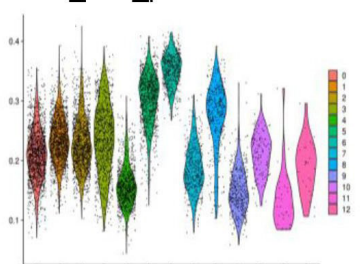

0123456789101112
Reactome_cell_cycle

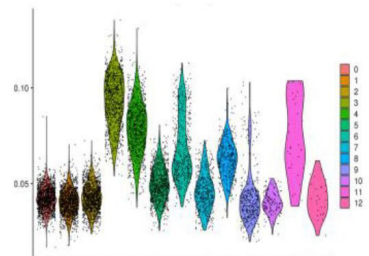

0123456789101112 $\begin{array}{lll}-0.4 & 0 & 0.4\end{array}$

Figure 2 Identification of human iPSC-derived CDCs differentiated on OP9-DL1 cells by single-cell RNA sequencing. (A) UMAP plots of human iPSC-derived HLA-DR ${ }^{+}$cells differentiated cells on OP9 and OP9-DL1 cells. Frequency of each cluster is shown. (B) Distribution of computationally predicted cell types determined by SingleR using the Novershtern Hematopoietic reference. (C) Heatmap displaying mean normalized expression of selected genes in each cluster. (D) Raindrop plots displaying mean normalized expression of selected genes in each cluster. (E) Single-cell enrichment scores of cDC1-related and monocyterelated genes shown in (D). (F) Violin plots of single-cell enrichment scores within each cluster. 
A

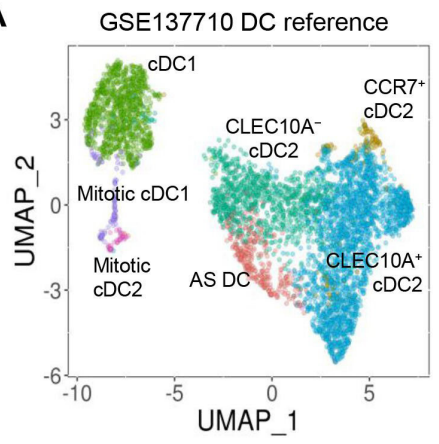

B

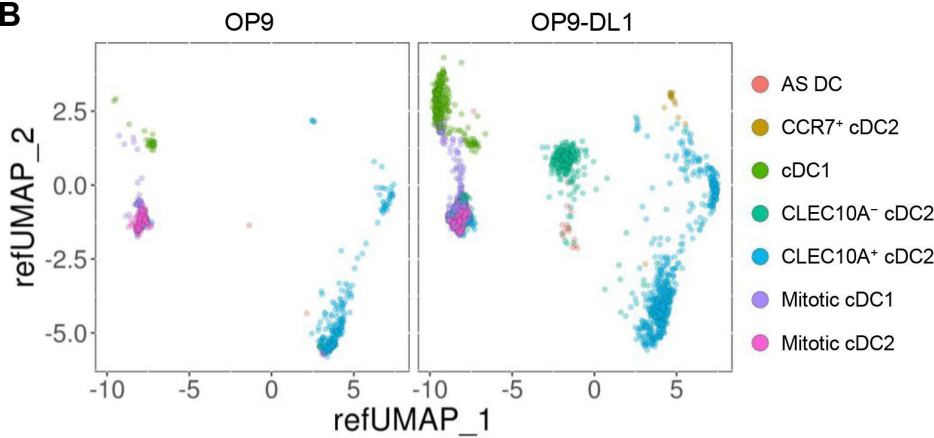

C

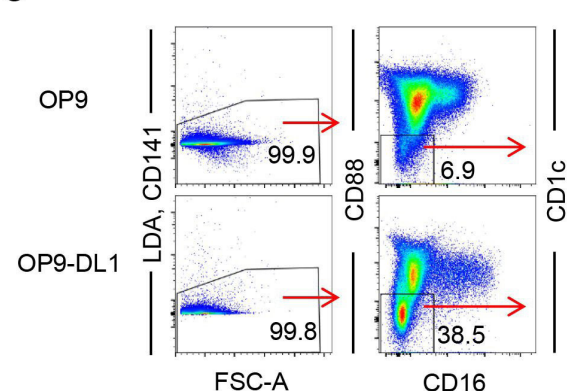

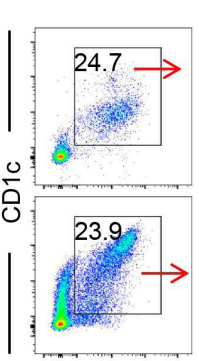

HLA-DR

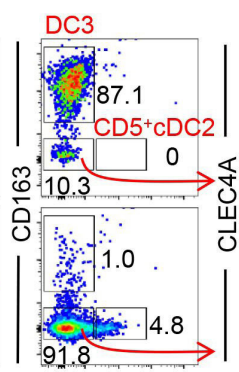

CD5
D

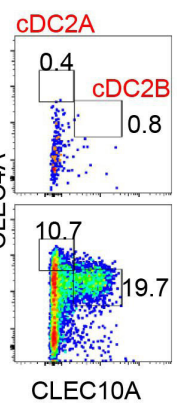

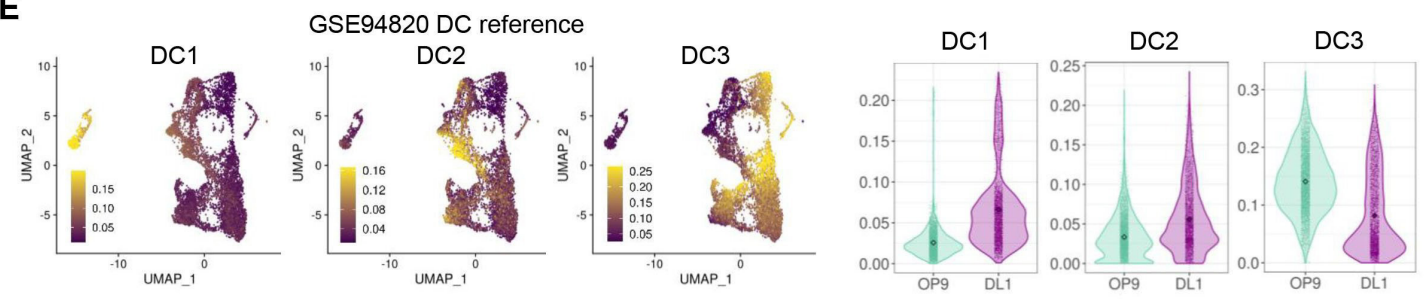

GSE132566 DC reference
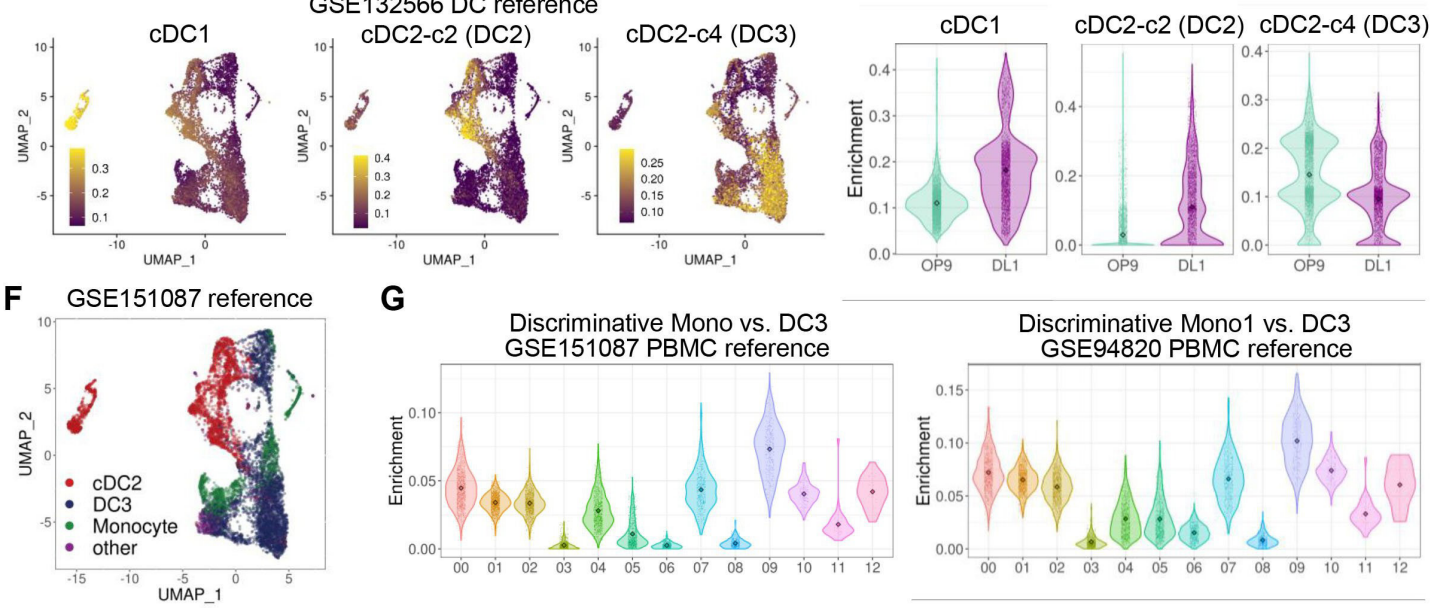

G
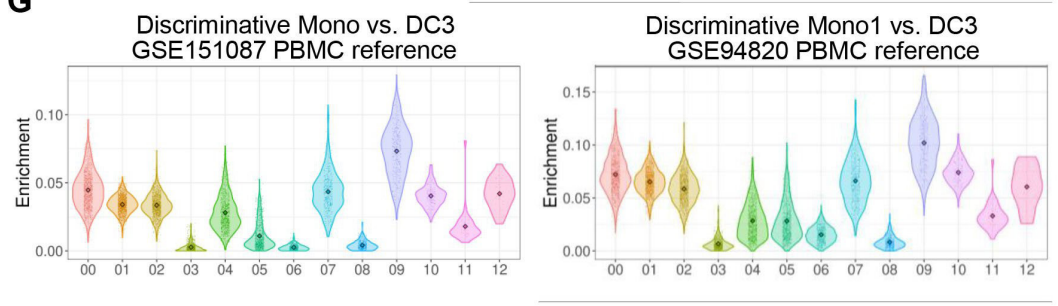

Figure 3 Heterogeneity of CD1c ${ }^{+}$DCs differentiated from human iPSCs. (A) UMAP plot of human Lin(CD3, CD56, CD19)

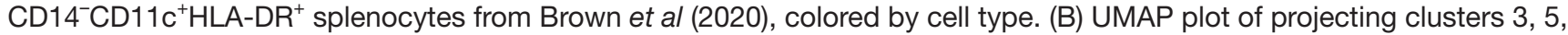
6 , and 8 from figure 2 of human iPSC-derived HLA-DR ${ }^{+}$cells differentiated on OP9 and OP9-DL1 cells projected onto human splenocyte atlas generated by Brown et al (2020). (C and D) Representative flow cytometric plots showing marker expression of human iPSC-derived cells on OP9 (upper) and OP9-DL1 (lower) feeder cells. Data shown are representative of two independent experiments. LDA, Live/Dead Aqua. (E) UMAP plots of single-cell enrichment scores using gene sets for specific DC subtypes derived from Villani et al (2017) (upper: Lin(CD3, CD56, CD19) $\left.{ }^{-} H L A-D R^{+} C D 11 c^{+} C D 14\right)$ ) and Dutertre et al (2019) (lower: cDC1: Lin(CD3, CD19, CD20) ${ }^{-} \mathrm{CD} 141^{+} \mathrm{CD} 14^{-} \mathrm{CD} 5^{-} \mathrm{CD} 123^{-} \mathrm{CD}^{-} \mathrm{c}^{-} \mathrm{HLA}-\mathrm{DR}^{+}$cells, cDC2-c2: $\mathrm{Lin}^{-} \mathrm{CD} 14^{-} \mathrm{CD} 1 \mathrm{c}^{+}$cells, cDC2-c4: $\mathrm{Lin}^{-} \mathrm{CD} 14^{+} \mathrm{CD} 1 \mathrm{c}^{+}$cells). Violin plots show cell type score distributions in human iPSC-derived HLA-DR ${ }^{+}$cells differentiated on OP9 and OP9-DL1 cells. (F) UMAP plot showing cell type predictions of total cells from figure 2 of human iPSC-derived HLA$\mathrm{DR}^{+}$cells differentiated on OP9 and OP9-DL1 cells derived from projection onto human PB CD16 ${ }^{-} \mathrm{CD} 123^{-} \mathrm{CD} 141^{-} \mathrm{CD} 14^{+/-} \mathrm{CD} 1 \mathrm{c}^{+}$ cell atlas generated by Bourdely et al (2019), colored by cell type. (G) Violin plots showing single-cell enrichment scores in each cluster from total cells in figure 2 using discriminative gene sets differentiating PB CD14 ${ }^{+}$CD16 $6^{-}$monocytes compared with DC3 cells. Gene signatures were derived from direct comparisons of annotated clusters by Bourdely et al (2019) (Mono vs DC3, left) and by Villani et al (2017) (Mono1 vs DC3, right). 
elevated in OP9 feeder cell group cells, with clusters C3, 5,6 , and 8 showing minimal DC3 enrichment (figure 3E and online supplemental figure 12D). Consistent with this, we found that enrichment scores using the gene set specific to DC3 cells determined from PB Lin(CD3/

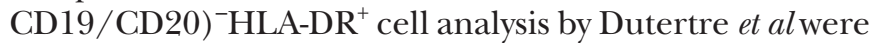
also elevated in OP9 feeder cell group cells (figure 3E). Furthermore, using a reference map generated from $\mathrm{PB}$ $\mathrm{CD} 16^{-} \mathrm{CD} 123^{-} \mathrm{CD} 141^{-} \mathrm{CD} 14^{+/-} \mathrm{CD} 1 \mathrm{c}^{+}$cells by Bourdely et al (online supplemental figure 13A), we inferred cell types and determined populations of DC3 and monocytes (online supplemental figure 13B,C). On projecting total cells from our scRNAseq data onto the Bourdely et al reference map and computationally inferring cell states, we found markedly increased DC3 cells in iPSC-DCs differentiated on OP9 cells compared with OP9-DL1 cells while moderate overlap between DC3s and monocytes was observed (figure 3F and online supplemental figure 13D). Of note, this reference map was generated without PB cDC1s; therefore, iPSC-derived CDC1 was annotated as CDC2 in the figure 3F likely due to their expression of $\mathrm{CD} 1 \mathrm{c}$ (figure 1D). Using gene sets differentiating monocyte populations from DC3 reported by Bourdely et al and Villani et al, we assessed single-cell enrichment scores and found there were differences of monocyte-related versus DC3-related gene expression among C0, 1, 2, 7, 9, 10 , and 12 (figure $3 \mathrm{G}$ ) initially annotated as monocytes (figure 2B).

Of note, we evaluated expression of plasmacytoid DC (pDC)-specific genes ${ }^{11} 20$ including GZMB, PTCRA, LILRA4, CLEC4C, and RUNX2, but did not identify any distinct cluster of pDCs (figure 2D). To confirm this, we used a multimodal reference atlas of the human PBMCs reported by Hao et al. ${ }^{49}$ We projected total cells in our scRNAseq data onto the previously annotated cells reported by Hao et $a l^{49}$ to generate a joint UMAP representation and infer immune cell states (online supplemental figure 14A). From this analysis, we confirmed there were no pDCs identified either on OP9 or OP9DL1 cells (online supplemental figure 14B,C). In agreement with this, flow cytometric analysis of iPSC-derived CD16 ${ }^{-} \mathrm{HLA}_{-} \mathrm{DR}^{+}$cells identified $\mathrm{CD} 123^{+} \mathrm{CD} 303^{+}$population, the majority of which, however, were not $\mathrm{CD} 45 \mathrm{RA}^{+} \mathrm{C}-$ D11 ${ }^{-} \mathrm{CX} 3 \mathrm{CR}^{-}{ }^{-} \mathrm{CD} 33^{-} \mathrm{pDCs}$ (online supplemental figure 15A). Some $\mathrm{CD} 123^{+} \mathrm{CD} 303^{+}$cells contained $\mathrm{CD} 45 \mathrm{RA}^{+} \mathrm{C}-$ $\mathrm{D} 33^{+} \mathrm{CX} 3 \mathrm{CR} 1^{+} \mathrm{CD}^{+}$cells resembling a subset, recently identified as pre-DCs (online supplemental figure 15B). ${ }^{44}$ Taken together, our findings demonstrate a critical role of Notch signaling in regulating developmental pathway of human cDCs.

\section{Notch signaling is required for differentiation of human iPSCs into CDC1s, CDC2s, AS-DCs but not DC3}

Having identified cDC1-like cells in human iPSC-derived HLA-DR ${ }^{+}$cells differentiated on OP9-DL1 but not OP9 cells by conventional flow cytometry and scRNAseq, we employed a loss-of-function approach using a $\gamma$-secretase inhibitor, DAPT,${ }^{20}$ to verify the role of Notch signaling in generation of cDC1s from human iPSCs. This inhibitor blocks the Notch signaling pathway by inhibiting the last proteolytic step before the release of Notch1 intracellular domain (NICD1), which is essential for Notch activation. Human iPSC-derived CD $34^{+}$cells were cultured on OP9 or OP9-DL1 cells in the presence or absence of DAPT, and iPSC-derived HLA-DR ${ }^{+}$cells were characterized by flow cytometry. We found that addition of DAPT markedly decreased the generation of cDC1-like cells and AS-DCs differentiated on OP9-DL1 cells (figure 4A and online supplemental figure 16). Furthermore, inhibition of Notch signaling substantially decreased cDC2 subsets CLEC4A ${ }^{\text {hi }}$ CLEC10A ${ }^{-}$(cDC2A), CLEC4A ${ }^{\text {lo- }}$ $\mathrm{CLEC10A}^{+}$(cDC2B), and $\mathrm{CD}^{+} \mathrm{CD}^{-} 163^{-}\left(\mathrm{CD}^{+}{ }^{+} \mathrm{cDC} 2\right)$ but increased $\mathrm{CD}^{-} \mathrm{CD} 163^{+} \mathrm{CD}^{+} \mathrm{c}^{+} \mathrm{HLA}^{-} \mathrm{DR}^{+}$DCs (DC3) (figure 4B). Thus, these results demonstrate that Notch signaling is required for in vitro differentiation of human iPSCs to cDC1-like cells, AS-DCs, cDC2As, cDC2Bs, and $\mathrm{CD}^{+}{ }^{+} \mathrm{cDC} 2 \mathrm{~s}$ but not DC3s.

\section{Notch-activated iPSC-derived CD $141^{+}{ }^{+}$CR $11^{+}$CLEC9A ${ }^{+}$DCs and human peripheral blood CDC1s display similar gene expression profile}

Previous studies have shown the generation of CD $141^{+} \mathrm{XCR}^{+}{ }^{+} \mathrm{DC}$ s from human iPSCs under feeder-free and xeno-free conditions without Notch signaling. ${ }^{19}$ Now that human iPSCs can be derived and maintained under feeder-free and xeno-free conditions, this approach might allow us to generate cDCs from human somatic cells under defined feeder-free and xeno-free conditions. In this protocol, human iPSCs were cultured with a cocktail of cytokines including GM-CSF, SCF, VEGF, BMP4, and IL-4 without xeno-components under defined conditions. In our hands, we added Flt3L to this protocol and found that human iPSCs differentiated into $\mathrm{CD} 141^{+} \mathrm{XCR} 1^{+} \mathrm{CLE}-$ $\mathrm{C} \mathrm{A}^{+} \mathrm{HLA}-\mathrm{DR}^{+}$cells (online supplemental figure $17 \mathrm{~A}-\mathrm{C}$ ). Of note, HLA-DR ${ }^{+}$cells differentiated from TiPSCs did not express T-cell receptor $\alpha / \beta$ (online supplemental figure 18).

At this point, we found cDC1-like cells expressing CD141, XCR1, and CLEC9A were generated from human iPSCs with or without xenogeneic feeder cells; however, similarities of gene expression between human iPSC-derived cDC1-like cells and peripheral blood cDC1s remain unknown. To further explore the effect of DL1-Notch signaling on in vitro human iPSC-derived DCs, we flowsorted $\mathrm{XCR}^{+}{ }^{+} \mathrm{CLEC} 9 \mathrm{~A}^{+} \mathrm{HLA}-\mathrm{DR}{ }^{+} \mathrm{CD} 11 \mathrm{c}^{+}$cells differentiated from on-feeder and feeder-free culture conditions (hereafter referred to as DL1-iPSC-cDC1s or FF-iPSCcDC1s, respectively), and interrogated their expression profiles by global mRNA sequencing (RNAseq). Peripheral blood XCR1 ${ }^{+}$CLEC9A $^{+} \mathrm{HLA}^{-} \mathrm{DR}^{+} \mathrm{CD} 11 \mathrm{c}^{+}$cells (PB-cDC1s) were used as a control.

To determine relationships between PB-cDC1s, DL1iPSC-cDC1s, and FF-iPSC-cDC1s, we performed hierarchical clustering of all 2748 differentially expressed genes (DEGs; fold change $>1.5$, fdr adjusted $\mathrm{p}$ value $<0.05$ ) identified between groups and visualized expression patterns 
A

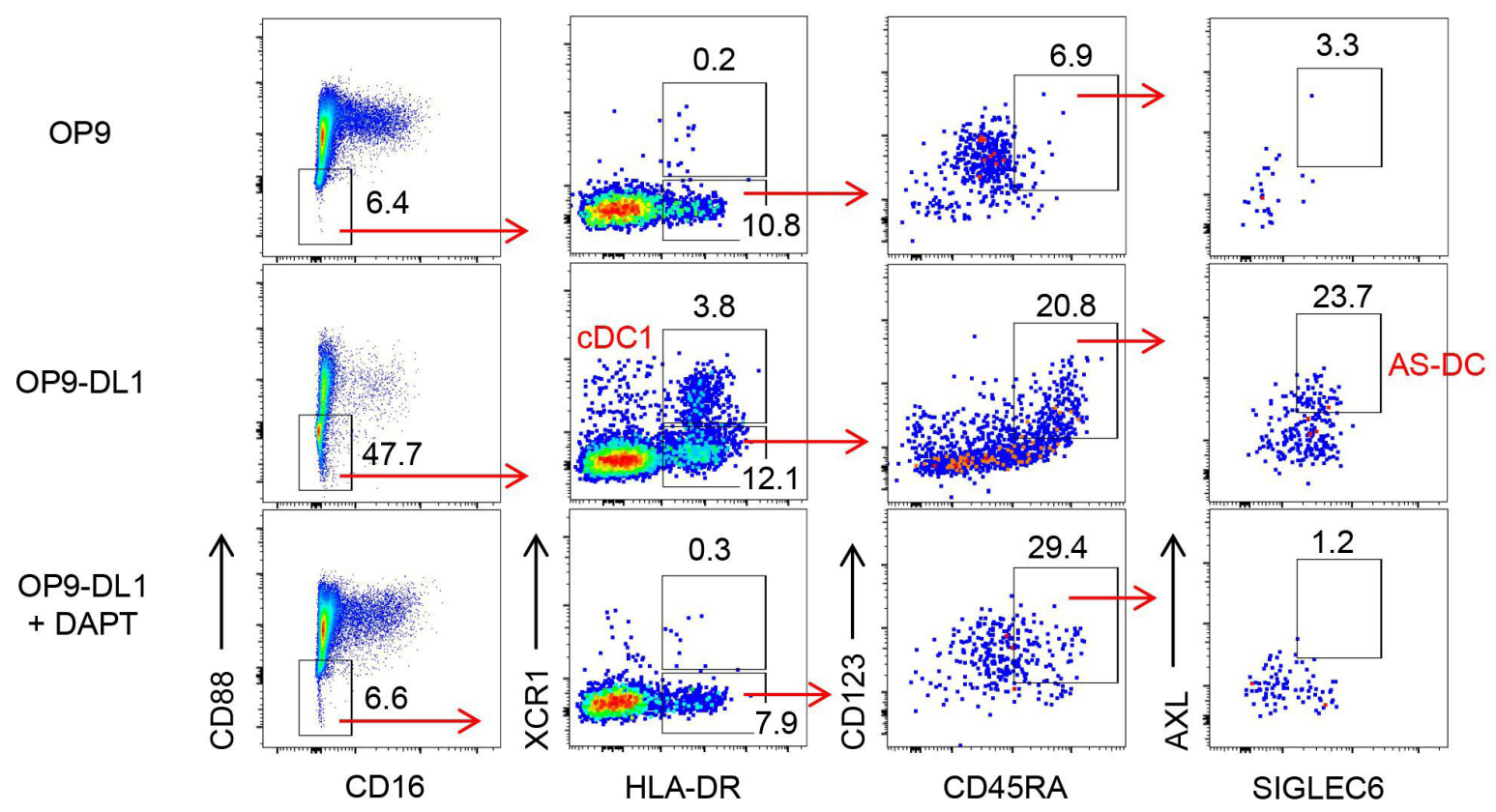

B
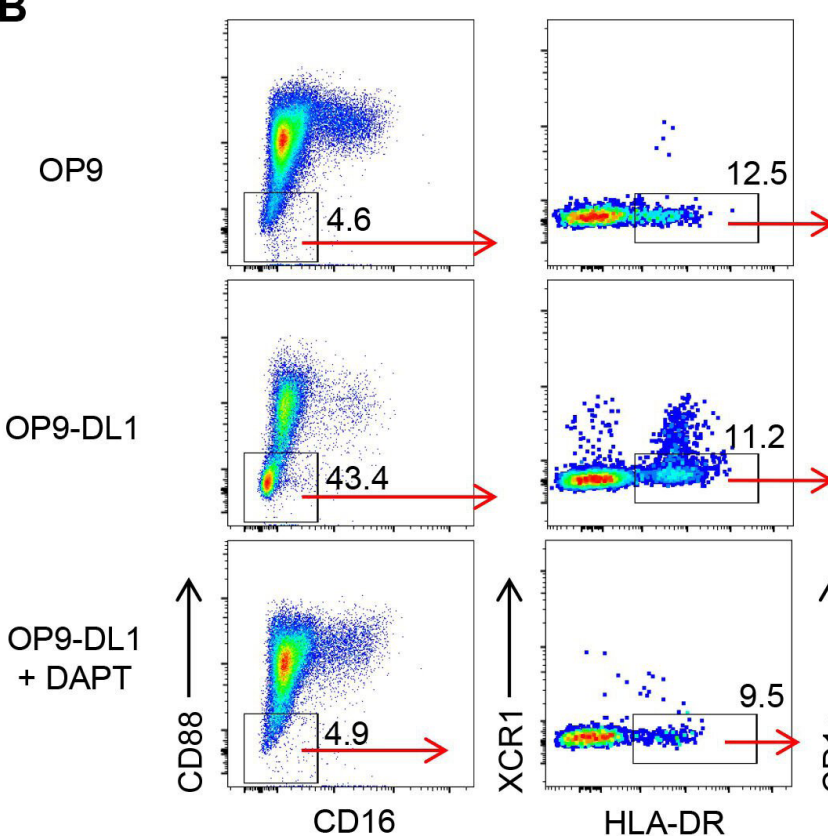

HLA-DR
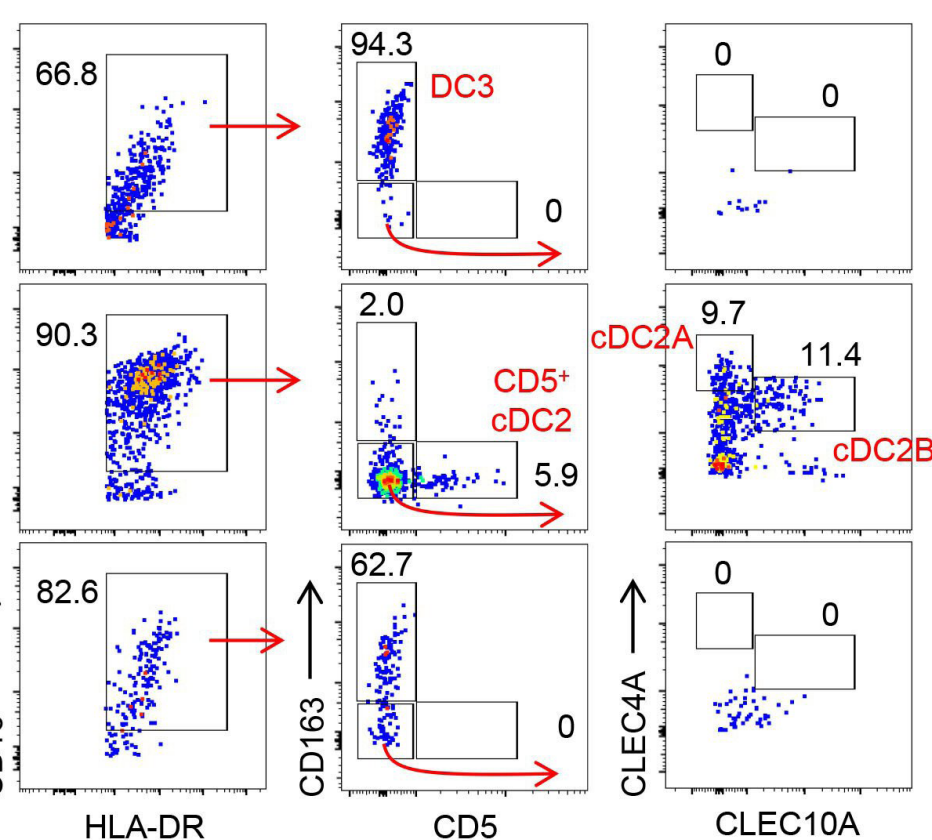

Figure 4 Notch signaling is required for generation of CDCs from human iPSCs. (A, B Human iPSCs were differentiated on OP9 or OP9-DL1 cells in the presence or absence of a $\gamma$-secretase inhibitor, $\mathrm{N}$-[N-(3,5-difluorophenacetyl)- L-alanyl]$S$-phenylglycine t-butyl ester (DAPT). The gating strategy and frequency of $X C R 1^{+} H L A-D R^{+} C D C 1 s$ and AS-DCs (A) and

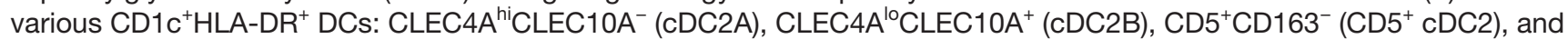
$\mathrm{CD}^{-}{ }^{-} \mathrm{CD} 163^{+}(\mathrm{DC} 3)(\mathrm{B})$ are shown.

using a dendrogram and a heat map (figure 5A). The clustering pattern indicates that the replicates of DL1iPSC-cDC1s and PB-cDC1s share similar transcriptional patterns. However, FF-iPSC-cDC1s are transcriptionally distinct from DL1-iPSC-cDC1s and PB-cDC1s. Similarly, PCA indicated that a major proportion of variation in transcriptional states across samples could be explained by the source of $\mathrm{CDC1}$ isolation and further demonstrated that PB-cDC1s are more closely related to DL1-iPSCcDC1s than to FF-iPSC-cDC1s (figure 5B). Concordance of DEGs between the three groups is represented by a Venn diagram (figure 5C). We found markedly more DEGs between FF-iPSC-cDC1s and DL1-iPSC-cDC1s (1678 DEGs) or PB-cDC1s (2200 DEGs) compared with between DL1-iPSC-cDC1s and PB-cDC1s (213 DEGs) (figure 5C). Volcano plots for FF-iPSC-cDC1s versus PB-cDC1s showed cDC1-related genes including CLEC9A, IDO1, XCR1, and $C A D M 1$ are significantly reduced while the expression of these genes was similar between DL1-iPSC-cDC1s and PB-cDC1s (figure 5D). We further evaluated DEGs 
A

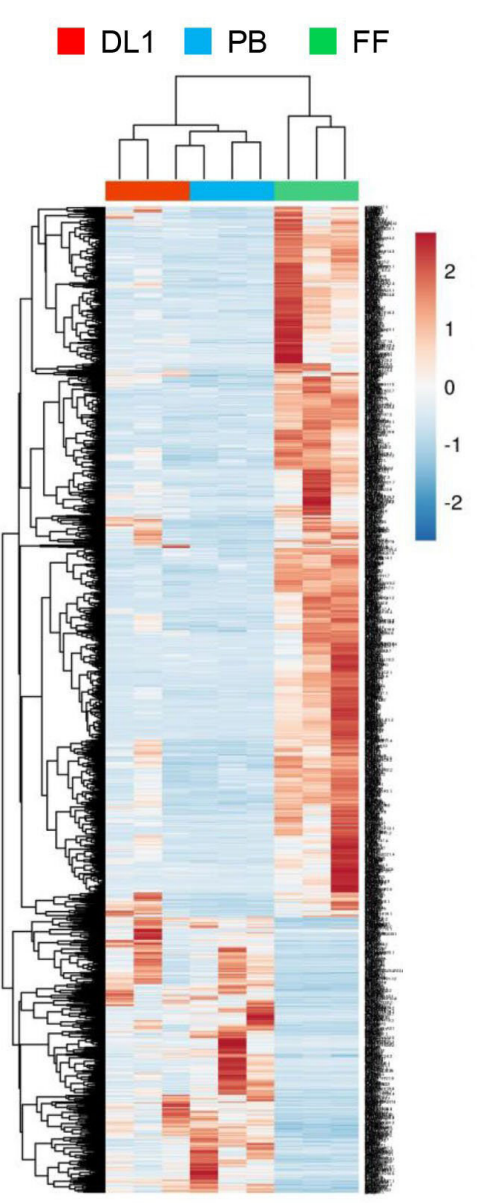

B

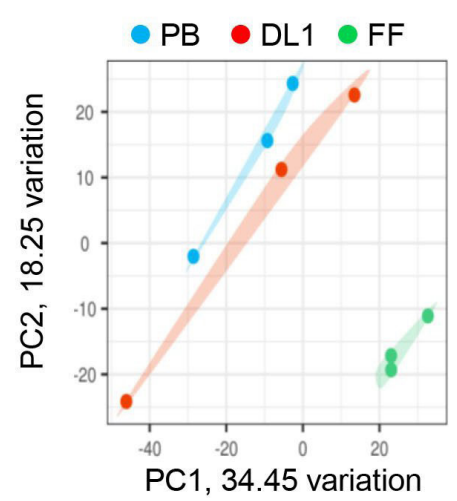

C

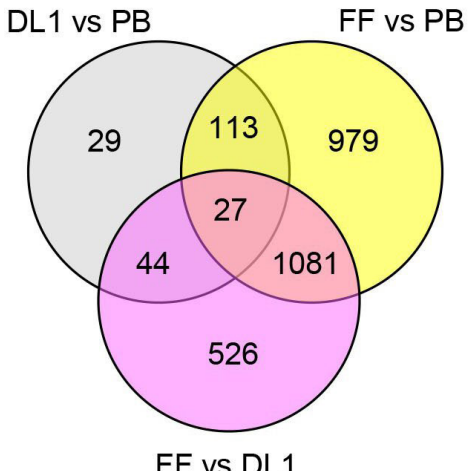

FF vs DL1
D

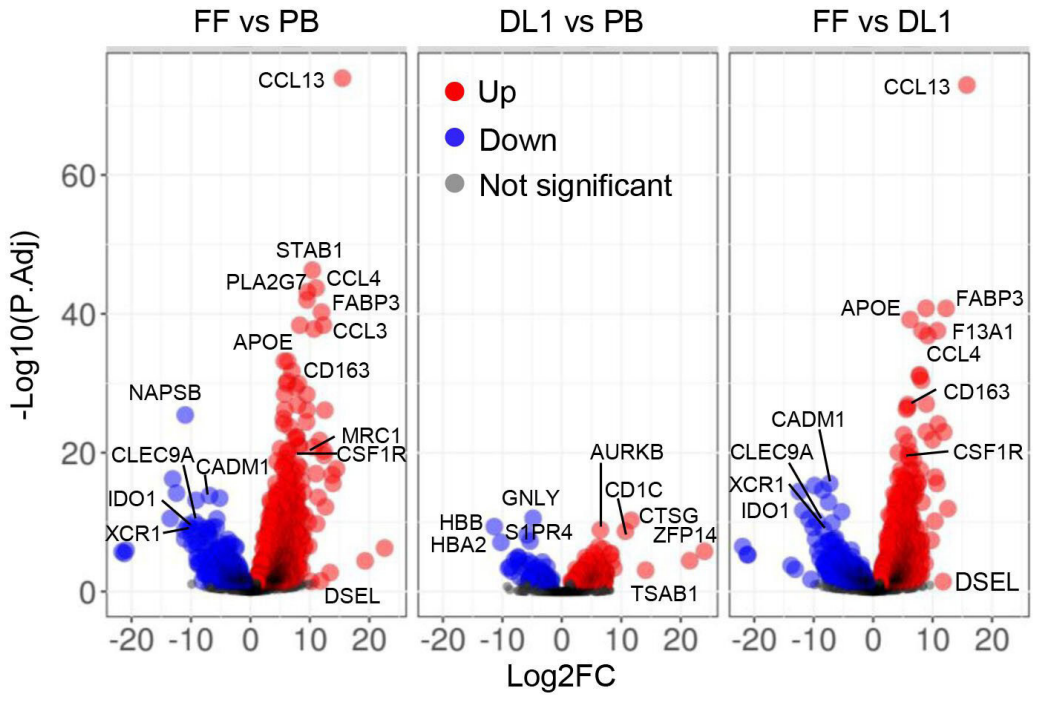

E

$\oslash \mathrm{PB} \square \mathrm{FF} \square \mathrm{DL} 1$

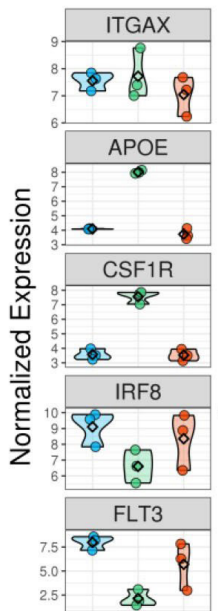

F

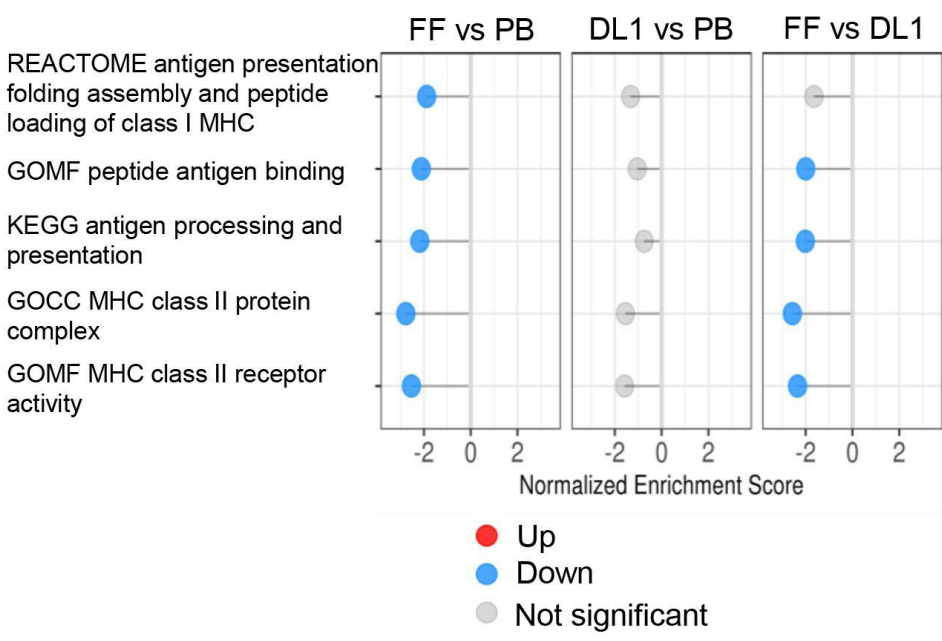

Figure 5 Notch-activated human iPSC-derived CD141 ${ }^{+} X C R 1^{+} C L E C 9 A^{+} D C s$ and human peripheral blood $C D C 1 s$ display similar gene expression profile. (A) Heat map representing genes differentially expressed between $X C R 1^{+} C L E C 9 A^{+} H L A-$ $\mathrm{DR}^{+} \mathrm{CD} 11 \mathrm{c}^{+}$cells differentiated on OP9-DL1 cells (DL1) or under feeder-free (FF) condition, or in peripheral blood (PB). (B) Principal component analysis (PCA). (C) Venn diagram of differentially expressed genes in DL1, FF, and PB $\mathrm{XCR} 1^{+}$CLEC9A ${ }^{+} \mathrm{HLA}-\mathrm{DR}{ }^{+} \mathrm{CD} 11 \mathrm{c}^{+}$cells. (D-F) Volcano plot (D), representative gene expression (E), and gene set enrichment analysis (GSEA) (F) of DL1, FF, and PB XCR1 $1^{+}$CLEC9A ${ }^{+} H L A-D R^{+} C D 11 c^{+}$cells.

between groups and found that although ITGAX gene, encoding for the CD11c protein, was similarly expressed in all groups, DL1-iPSC-cDC1s and PB-cDC1s expressed higher levels of cDC1-related and MHC-related genes than FF-iPSC-cDC1s (figure 5E). In contrast, genes associated with monocytes/macrophages such as ITGAM, CD163, APOE, FABP4, MRC1, and CSF1R were higher in FF-iPSC-cDC1s. Accordingly, DL1-iPSC-cDC1s and 
PB-cDC1s were notable for high levels of pathways related to MHC class II, and antigen processing and presentation compared with FF-iPSC-cDC1s (figure 5F). Together, DL1-Notch signaling allows human iPSCs to differentiate to peripheral blood cDC1-like cells in vitro.

\section{Phagocytic, T-cell proliferative, and cytokine-producing capacities of DL1-iPSC-DCs}

To gain insights into the function of iPSC-cDCs, we evaluated the capacity of iPSC-DCs differentiated on OP9DL1 feeder cells to phagocytose antigen and activate T cells. The phagocytic capacity of human iPSC-DCs was measured by amounts of cellular absorption of pHrodo zymosan particles, which become fluorescent once at acidic $\mathrm{pH}$ such as in phagosomes. We found that pHrodo zymosan particles were efficiently phagocytosed by human iPSC-derived cells (figure 6A), and iPSC-derived cDC1s, cDC2As, cDC2Bs, and DC3s demonstrated comparable phagocytic ability (figure 6B-D). We also evaluated the phagocytic ability of FF-iPSC-cDC1s and found that many FF-iPSC-cDC1s were not viable after phagocytosis of zymosan particles (online supplemental figure 19A,B).

We next tested whether human iPSC-DCs could enhance proliferation of $\mathrm{T}$ cells. To this end, we co-cultured human iPSC-DCs with isolated $\mathrm{T}$ cells from the same donor in the presence of MHC class I or II peptides specific to various viruses and IL-2. Human iPSC-DCs differentiated on OP9-DL1 cells facilitated proliferation of $\mathrm{CD}^{+}$and $\mathrm{CD}^{+} \mathrm{T}$ cells in the presence of MHC class I and II peptides, respectively (figure 7A). We found that both proliferating $\mathrm{T}$ cells effectively downregulated CD62L and CD45RA, and upregulated CD25 (figure 7B). Small populations of proliferating $\mathrm{CD} 8^{+}$and $\mathrm{CD} 4^{+} \mathrm{T}$ cells maintain the expression of CD45RA, CD62L, and CD27, suggesting less-differentiated $\mathrm{T}$ cells (figure 7C). Furthermore, flow-sorted CLEC9A ${ }^{+}$DL1-iPSC-cDC1s induced proliferation of $\mathrm{CD} 8^{+} \mathrm{T}$ cells in the presence of MHC class I peptides (figure 7D).

Lastly, we evaluated cytokine-producing capacity of iPSC-DCs. DL1-iPSC-cDC1s, cDC2As, cDC2Bs, and OP9iPSC-DC3 produced IL-12 and TNF- $\alpha$ on stimulation with TLR agonists, poly(I:C), R848, LPS, and CpG (figure 8A). IFN- $\lambda$ was secreted by DL1-iPSC-cDC1s while negligible IFN- $\alpha$ was produced by either OP9-iPSC-DCs or DL1iPSC-DCs (figure 8B and C). Collectively, human iPSCs could differentiate into various functional $\mathrm{cDC}$ subsets in vitro.

\section{DISCUSSION}

In this study, we demonstrated generation of cDC-like cells from human iPSCs. Key results described herein include (1) Notch-signaling dependent efficient in vitro differentiation of human iPSCs into $\mathrm{CD} 141^{+} \mathrm{XCR} 1^{+} \mathrm{CLE}-$ $\mathrm{C} \mathrm{A}^{+} \mathrm{HLA}-\mathrm{DR}^{+}$cells resembling PB cDC1s; (2) generation of various $\mathrm{cDC}$ subsets but not pDCs from human iPSCs; (3) identification of the heterogeneity of human iPSCderived cDCs including cDC2A, cDC2B, $\mathrm{CD}^{+} \mathrm{CDC}^{+}$,
DC3, and AS-DC subsets (online supplemental figure 20); and (4) phagocytic, T cell proliferative, and cytokineproducing capacities of human iPSC-derived cDC-like cells.

Recent evidence suggests Notch-signaling dependent differentiation of cDC1s from CD34 ${ }^{+}$HSCs. ${ }^{20} 21$ Our study is in line with this, and further demonstrated efficient generation of cDC1-like cells as well as various cDCs including cDC2As, cDC2Bs, CD5 ${ }^{+}$cDC2s, and AS-DCs from human iPSC-derived CD34 ${ }^{+}$HPCs. Although these two recent studies have shown the generation of IFN- $\alpha$-producing pDCs from human CD34 ${ }^{+}$HSCs in co-culture with OP9 cells, we did not identify pDCs derived from human iPSCs co-cultured with either OP9 or OP9-DL1. The majority of $\mathrm{CD} 123^{+} \mathrm{CD} 303^{+}$cells in both OP9 and OP9-DL1 feeder cells expressed myeloid markers such as CD11c, CD33, and/or CX3CR1. Consistent with this, IFN- $\alpha$ production was not detected on stimulation with TLR agonists including CpG. Additional work is needed to better understand the molecular and cellular mechanisms underlying the development of pDCs from human iPSCs.

High-dimensional, single-cell RNA expression analysis confirmed the generation of CDC1s and also probed the diversity of $\mathrm{CD}_{1 \mathrm{c}^{+}}$DCs in human iPSC-derived cells. We found that human iPSCs could differentiate into cDC2As expressing CLEC4A, RUNX3, LTB, IL22RA2, and IDO1, recently identified in human spleen as well as canonical cDC2Bs expressing CLEC1OA and FCER1A. Furthermore, human iPSC-derived DCs projected onto the reference human splenocyte map ${ }^{33}$ confirmed the generation of cDC2As. Notably, human iPSC-derived cDC2As were only identified on OP9-DL1 but not on OP9 cells, suggesting a potential role of Notch signaling in generation of cDC2As as well as cDC1s. These findings are congruent with increased expression of genes related to Notch signaling in cDC2As in mouse and human spleen. ${ }^{33}$ Evidence showed that cDC2As could be isolated from human spleen but not peripheral blood. ${ }^{33}$ This suggests that Notch signaling-mediated differentiation of human iPSCs allows us to generate primary cDC that may not be obtained from leukapheresis.

Our loss-of-function approach with a $\gamma$-secretase inhibitor for blocking Notch signaling provided mechanistic insight into the differentiation of other $\mathrm{CD} 1 \mathrm{c}^{+} \mathrm{DC}$ subsets. Addition of a $\gamma$-secretase inhibitor in an OP9-DL1 feeder system substantially decreased the frequency of cDC2Bs and $\mathrm{CD}^{+}{ }^{\mathrm{CDC}} 2 \mathrm{~s}$, suggesting that Notch signaling may also have an important role in differentiation of CDC2Bs and $\mathrm{CD}^{+}{ }^{+} \mathrm{cDC} 2 \mathrm{~s}$. In contrast, DC3s were differentiated either on OP9 or OP9-DL1 feeder cells, and increased with a $\gamma$-secretase inhibitor. This indicates that development of DC3 subset is independent of that of cDC1s and cDC2s in line with a recent work showing that DC3s do not differentiate via cDC-restricted common DC precursor (CDP). ${ }^{34}{ }^{45}$ Moreover, our results of increased frequency of CDP-derived DCs but decreased frequency of DC3s on OP9-DL1 feeder cells suggest that Notch signaling 
A

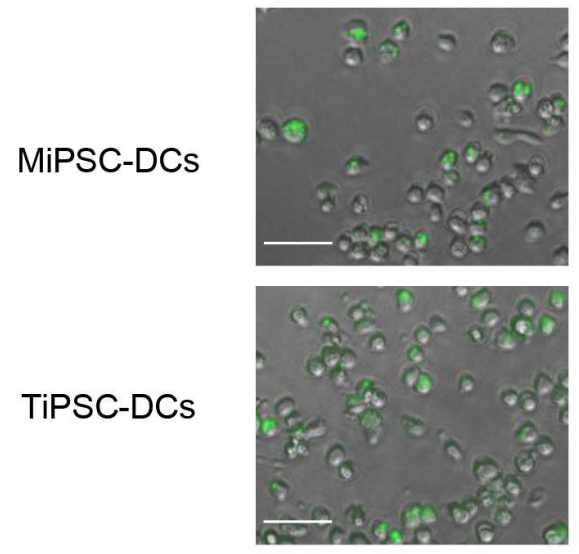

B

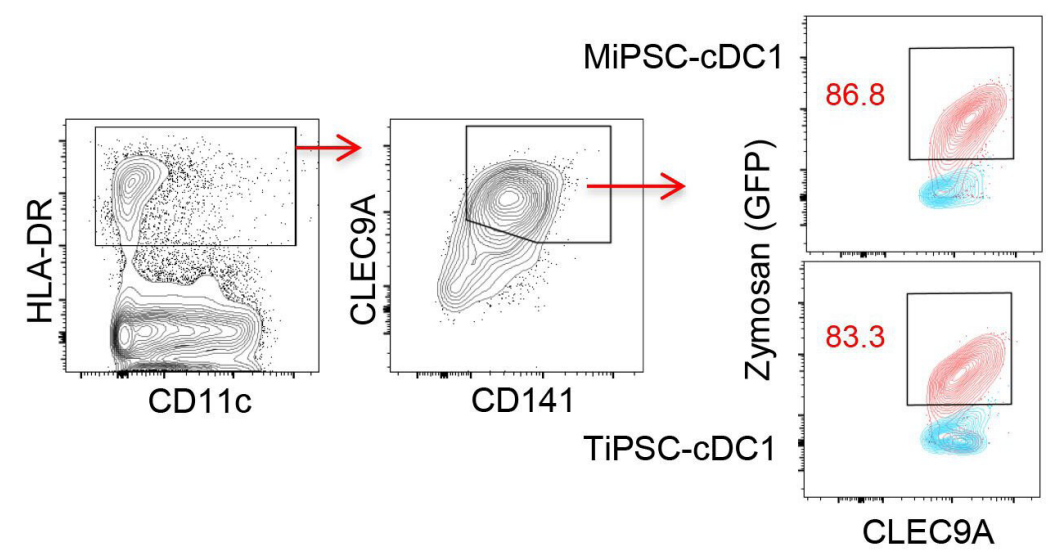

C

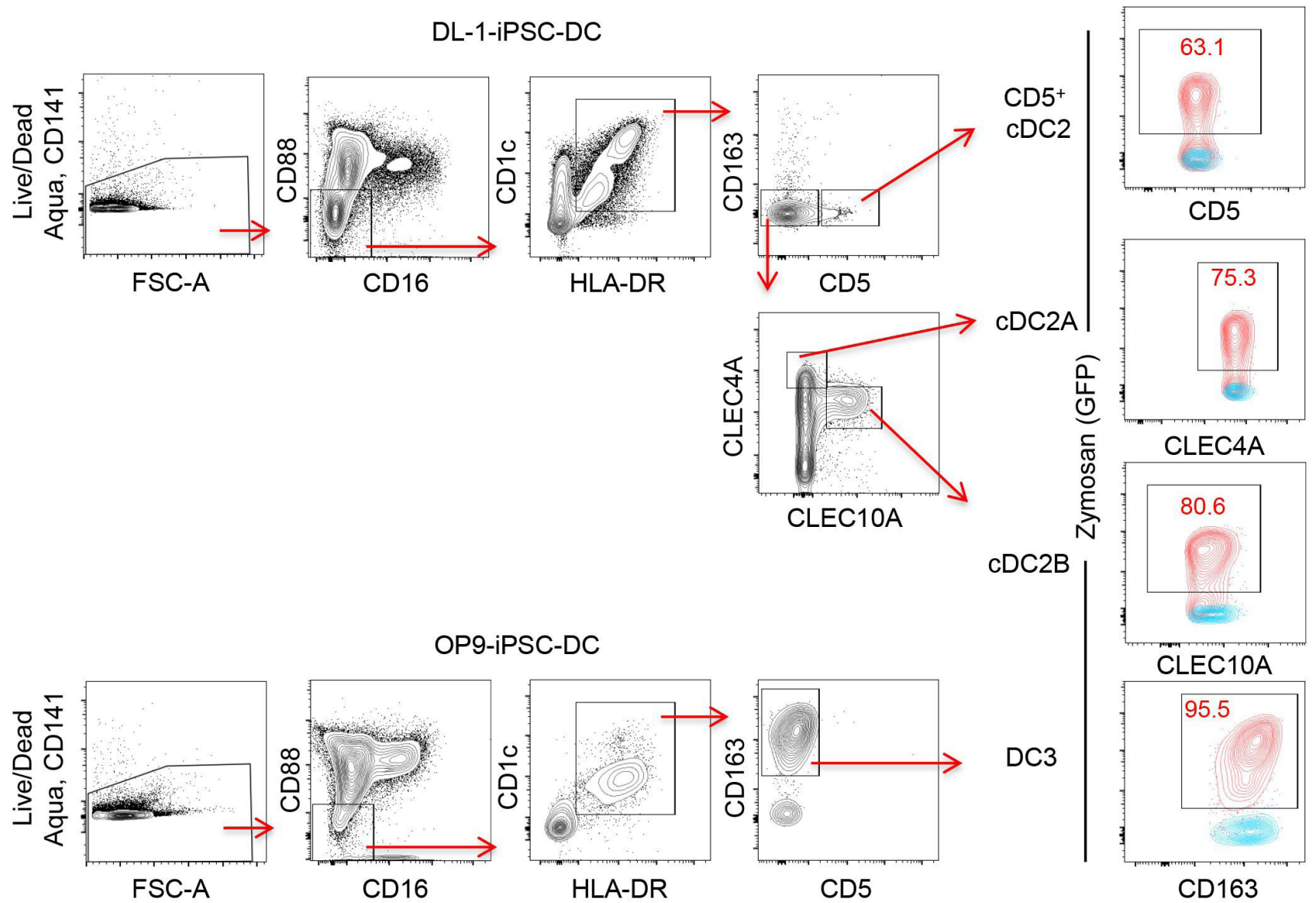

Figure 6 Phagocytic capacity of human iPSC-derived DCs. (A) Representative pictures of human iPSC-derived cells in coculture with pHrodo zymosan particles. MiPSC, monocyte-derived iPSC; TiPSC, T cell-derived iPSC (scale bar, $50 \mu \mathrm{m})$. (B,

C) Representative histogram showing zymosan expression in human iPSC-derived cDC1s (B), CD5 ${ }^{+}$cDC2s, cDC2As, cDC2Bs $(C)$, and DC3s (D) differentiated on OP9-DL1 (B, C) or OP9 cells (D). Data shown are representative of two independent experiments.

orchestrates the reciprocal development of these two distinct DC lineages. Nonetheless, data presented herein underscore that human iPSCs offer unique advantages for developmental studies. ${ }^{50}{ }^{51}$ More work is needed to delineate the mechanisms underlying the potential lineage decisions of cDC2s and DC3s.
Recent advances in single-cell molecular profiling have made it possible to identify various rare DC subsets including AS-DCs in peripheral blood. ${ }^{3644}$ The use of reference dataset relevant to the study of interest also improves the accuracy of cell-type classification and allows cell-type discovery in the sample without enrichment 
A
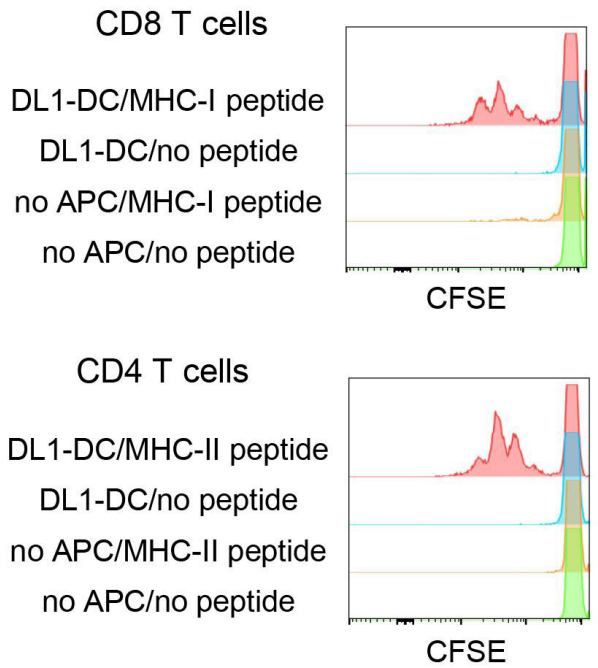

B
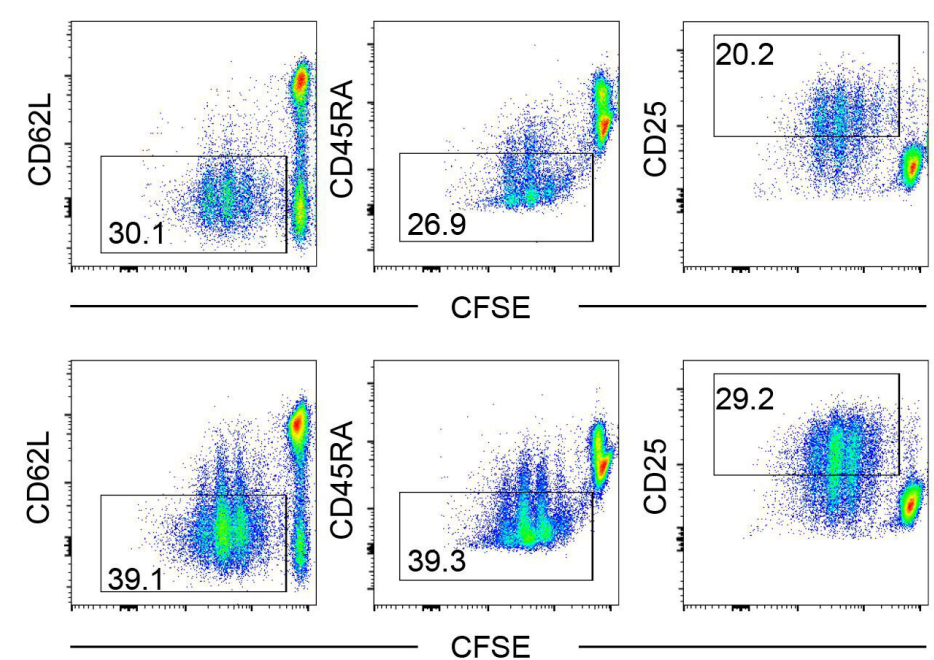

C
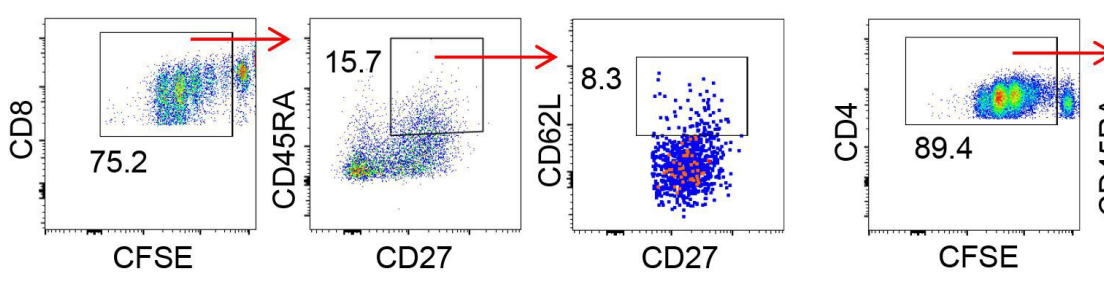

CFSE

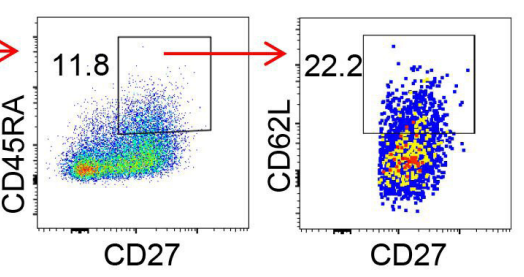

D
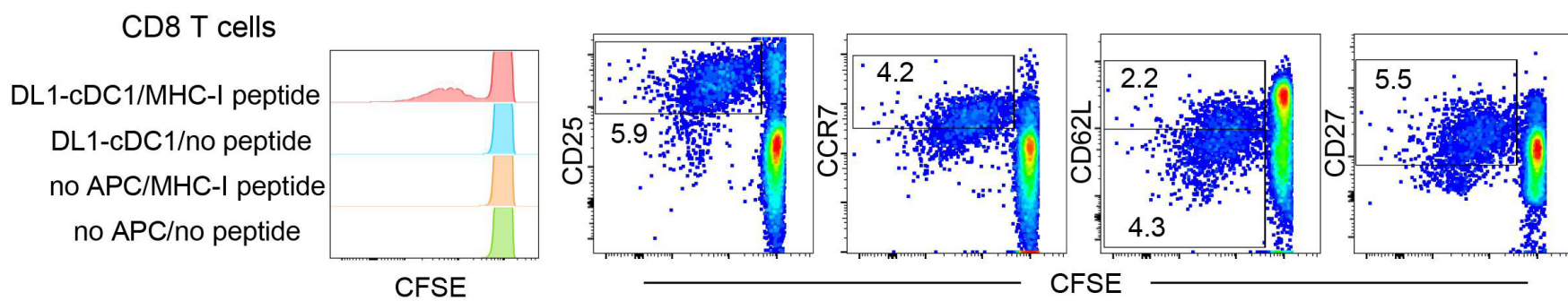

CFSE

Figure 7 T-Cell proliferative capacity of human iPSC-derived DCs. (A) Representative histogram showing CFSE expression in peripheral blood T cells co-cultured with or without DL-1-iPSC-DCs in the presence or absence with MHC class I or II peptides in vitro. $(B, C)$ Representative flow cytometric plots showing CFSE-labeled peripheral blood T cells from (A). (D) Representative histogram and flow cytometric plots showing CFSE expression in peripheral blood T cells co-cultured with or without DL1 -iPSC-CLEC9A ${ }^{+}$DCs in the presence or absence with MHC class I peptides in vitro. Data shown are representative of two independent experiments.

of rare cell types prior to scRNAseq. ${ }^{33}$ In this study, projecting our scRNAseq data onto the reference human splenocyte atlas helped identify AS-DC and cDC2As as well as confirm the presence of DC3 in human iPSCs-derived $\mathrm{HLA}_{-\mathrm{DR}}^{+}$cells. In addition, we found gene-expression profiling was necessary to determine a cell type in this study. Human iPSCs differentiated under feeder-free and xeno-free conditions without Notch signaling expressed cDC1 markers; however, bulk RNAseq analysis revealed that the transcriptomic landscape of $\mathrm{CD} 141^{+} \mathrm{XCR} 1^{+-}$ CLEC9A $^{+}$HLA-DR ${ }^{+}$cells generated under this condition markedly differed from DL1-iPSC-cDC1s and PB-cDC1s. Collectively, our study illustrates the significance of combining omics approaches and systems biology with conventional flow cytometric analysis in interrogating the heterogeneity and development of human iPSC-derived myeloid cells.

Our study has some limitations. More work is warranted to determine the function of each human iPSC-derived DC subset. For example, cDC1s specialize in crosspresentation and priming $\mathrm{CD}^{+} \mathrm{T}$ cells for tumors and pathogens that do not directly infect $\mathrm{DCs}^{52-54}$; however, cross-priming capacity of human iPSC-derived cDC1s remains unknown. Our study is limited to assessing in vitro functions of iPSC-derived cDCs. Although subsets of iPSC-derived cDCs expressed CCR7, their migratory potential remains elusive. Similarly, whether iPSC-derived cDCs exhibit the ability to phagocytose tumor antigens in the immunosuppressive tumor microenvironment remains an open question. We found DL1-iPSC-cDC1s 
A

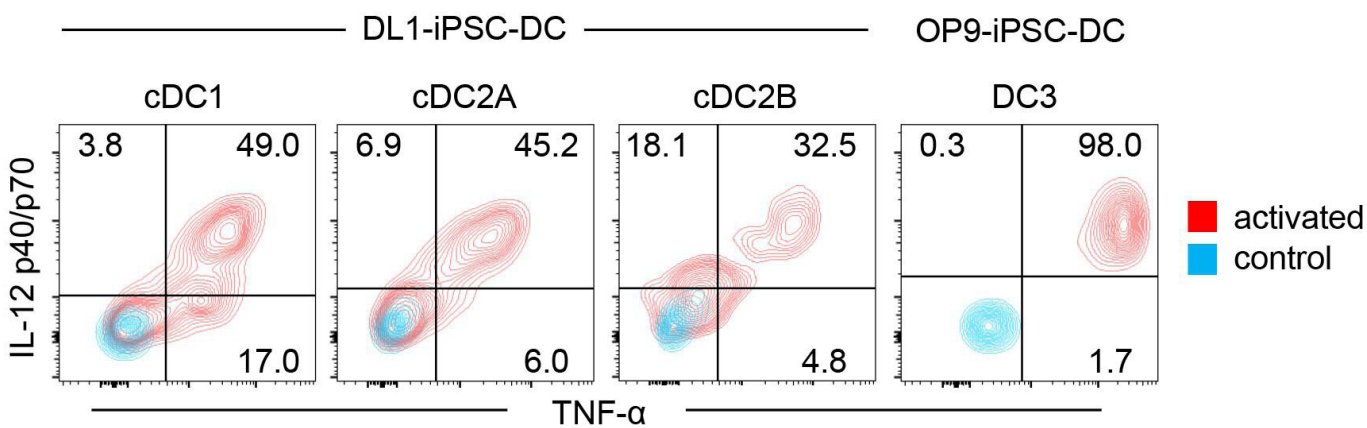

B
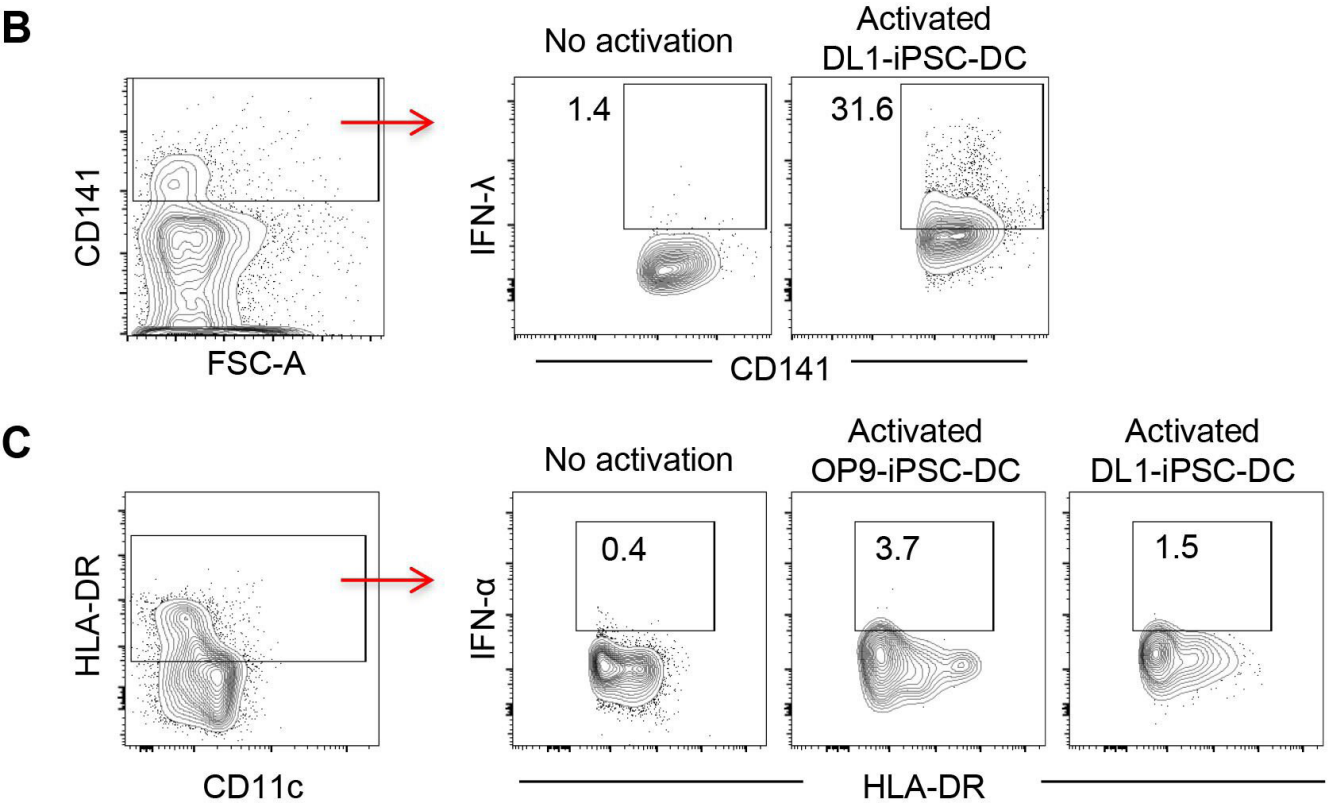

Figure 8 Cytokine-producing capacity of human iPSC-derived DCs. (A-C) IL-12p40/p70 (A), TNF- $\alpha$ (A), IFN- $\lambda$ (B), and IFN- $\alpha$ (C) production on stimulation with TLR agonists, poly(I:C), R848, LPS, and CpG by human iPSC-derived DCs differentiated

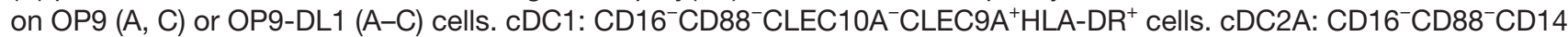

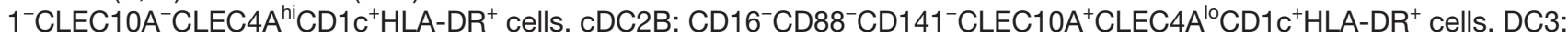
$\mathrm{CD}^{-} 6^{-} \mathrm{CD} 88^{-} \mathrm{CD} 141^{-} \mathrm{CD} 163^{+} \mathrm{CD} 1 \mathrm{C}^{+} \mathrm{HLA}-\mathrm{DR}^{+}$cells. Data shown are representative of two independent experiments.

expressed CD1c as observed in $\mathrm{CD} 141^{+}$cDC1s differentiated from human $\mathrm{CD} 34^{+}$cord blood progenitors in vitro and in Flt3L-induced CD141 ${ }^{+}$cDC1s in vivo. ${ }^{20} 4243$ Developmental process from double-positive $\mathrm{CD}_{1} \mathrm{c}^{+} \mathrm{CD} 141^{+}$ cDC1s to $\mathrm{CD} 141^{+}$cDC1s remains unknown. Our findings suggest a role of Notch signaling in differentiation of $\mathrm{CD}^{+}$cDC2s and AS-DCs on flow cytometric analyses. However, confirmation of this was limited due to the rarity of this subset in our scRNAseq data. Expression of CD5, AXL, and SIGLEC6 has been found in DC precursors, ${ }^{44}$ and evaluation of DC differentiation at different stages of human iPSC-derived cells might elucidate ontogeny and developmental pathway of $\mathrm{CD}^{+}{ }^{+} \mathrm{cDC} 2 \mathrm{~s}$ and AS-DCs. Our scRNAseq analyses and others have identified the presence of "mitotic DCs" with higher expression of cell cyclerelated genes in human iPSC-derived DCs and spleen, ${ }^{33}$ respectively. However, it remains unclear whether this cell population maintains the ability to proliferate. We aimed to generate cDC1s from human iPSCs and used Flt3L which is critical to development of DCs. Although the scope of this study was limited to determining Notch signaling for the development of cDC1s, one future area of investigation is to decipher growth factor requirements for the development of CDC1s as well as other DC subsets from human iPSCs. Lastly, potential tumorigenicity of iPSC-derived cDCs needs to be evaluated before this approach is clinically translated in the clinic.

Despite promising immunogenicity and favorable safety profiles, DC-based immunotherapies have elicited only limited clinical responses. ${ }^{57}$ One possible explanation for this is that DCs differentiated from peripheral blood monocytes in the presence of GM-CSF and IL-4 have less adjuvant capacity and immune-activating properties such as antigen-presentation capabilities due to lower MHC molecule expression compared with bona fide DC subsets. ${ }^{57}$ Clinical efforts using naturally circulating primary DCs are underway although they are relatively scarce. ${ }^{7}$ While clinical translational potential of iPSC-cDCs remains to be determined, one possible way to use them would be intratumoral injection. The frequency of cDC1s 
in the tumor correlates with prognosis and response to anti-PD-1/PD-L1 therapy, ${ }^{14}$ and in situ injection or mobilization of cDCs enhances response to anti-PD-1/PD-L1 therapy and immunogenicity of radiotherapy in preclinical models and patients. ${ }^{55-59}$ Regarding this, we have recently reported antitumor efficacy of multimodal intralesional therapy with in situ delivery of mouse iPSC-DC and radiation therapy against preclinical models of breast cancer resistant to anti-PD-L1 therapy. ${ }^{56}$ Future studies with humanized mouse models where MHC is matched between human iPSC-DCs and engrafted PBMCs or $\mathrm{CD}^{+} 4^{+}$cells are expected to provide insight into in vivo therapeutic efficacy of human iPSC-DCs compared with bona fide and monocyte-derived DCs. Considering the clinical application of human iPSC-derived cDCs, the use of recombinant human delta-like $1-\mathrm{Fc}$ antibody ${ }^{60}$ might provide the opportunity to generate cDCs from human iPSCs under feeder- and xeno-free conditions.

In summary, we reported in vitro methods to efficiently differentiate human iPSCs to $\mathrm{CD} 141^{+} \mathrm{XCR}^{+}{ }^{+} \mathrm{CLEC} 9 \mathrm{~A}^{+}$ DCs resembling PB-cDC1s. Loss-of-function and gain-offunction studies unraveled the role of Notch signaling in regulating generation of cDCs from human iPSCs. Singlecell profiling of human iPSC-derived DCs confirmed a cluster expressing high levels of CDC1 signature and revealed the heterogeneity of $\mathrm{CD}^{+} \mathrm{c}^{+} \mathrm{DCs}$ that warrants further investigation. Our work provides a solid foundation for the future development of personalized treatment with unlimited numbers of autologous $\mathrm{CD} 141^{+} \mathrm{XCR} 1^{+-}$ CLEC9A $^{+}$DCs from human iPSCs in the clinic.

\section{Author affiliations}

${ }^{1}$ Center for Immunotherapy, Roswell Park Comprehensive Cancer Center, Buffalo, New York, USA

${ }^{2}$ Department of Obstetrics and Gynecology, Akita University Graduate School of Medicine School of Medicine, Akita, Japan

${ }^{3}$ Department of Biostatistics and Bioinformatics, Roswell Park Comprehensive Cancer Center, Buffalo, New York, USA

${ }^{4}$ Department of Surgery, Shinshu University Graduate School of Medicine School of Medicine, Matsumoto, Nagano, Japan

${ }^{5}$ Department of Pathology, Roswell Park Comprehensive Cancer Center, Buffalo, New York, USA

${ }^{6}$ Department of Immunology, Roswell Park Comprehensive Cancer Center, Buffalo, New York, USA

${ }^{7}$ Department of Surgical Oncology, Roswell Park Comprehensive Cancer Center, Buffalo, NY, USA

${ }^{8}$ Department of Surgery, University of Southern California, Los Angeles, CA, USA

\section{Twitter Fumito Ito @lab_ito}

Acknowledgements We acknowledge Prometheus Laboratories Inc. for kindly providing recombinant human IL-2 and BioRender.com for illustrations. We thank Drs. Anm Nazmul Khan, Takayoshi Yamauchi, and Kumiko Iwabuchi (Roswell Park Comprehensive Cancer Center) for technical assistance.

Contributors KM designed and performed experiments, analyzed data, and wrote the manuscript. ML contributed the bioinformatics analyses and revised the article. RK, SM, and TO performed experiments, and revised the article. KK performed pathologic examination of tissue samples and revised the article. SL supervised the bioinformatics analyses and revised the article. Fl developed the concept, analyzed data, managed the project, coordinated author activities, revised the article, provided final approval of the version to be submitted, and is responsible for the overall content as the guarantor,
Funding This work was supported by National Cancer Institute (NCl) grant P30CA016056 involving the use of Roswell Park's Flow and Image Cytometry, Bioinformatics, and Genomic Shared Resources. This work was supported by the Melanoma Research Alliance and the Sarcoma Foundation of America (FI), Uehara Memorial Foundation (T0), and National Cancer Institute (NCl) grant, U24CA232979 (ML and SL), K08CA197966, and R01CA255240-01A1 (FI).

Competing interests None declared.

Patient consent for publication Consent obtained directly from a healthy volunteer

Ethics approval This study involves human participants and was approved by the Institutional Review Board (IRB) of Roswell Park Comprehensive Cancer Center (protocol \# BDR072916) and the University at Buffalo/Roswell Park Stem Cell Research Oversight (SCR0) Committee (protocol \#17-001-RP), and has been performed in accordance with the ethical standards of the responsible committee on human experimentation and with the Helsinki Declaration. An IRB-approved written informed consent was obtained from a healthy donor for being included in the study. Participants gave informed consent to participate in the study before taking part.

Provenance and peer review Not commissioned; externally peer reviewed.

Data availability statement Data are available on reasonable request. All data derived from RNA-seq and scRNA-seq studies are deposited at NCBI Gene Expression Omnibus under series accession GSE193488.

Supplemental material This content has been supplied by the author(s). It has not been vetted by BMJ Publishing Group Limited (BMJ) and may not have been peer-reviewed. Any opinions or recommendations discussed are solely those of the author(s) and are not endorsed by BMJ. BMJ disclaims all liability and responsibility arising from any reliance placed on the content. Where the content includes any translated material, BMJ does not warrant the accuracy and reliability of the translations (including but not limited to local regulations, clinical guidelines, terminology, drug names and drug dosages), and is not responsible for any error and/or omissions arising from translation and adaptation or otherwise.

Open access This is an open access article distributed in accordance with the Creative Commons Attribution Non Commercial (CC BY-NC 4.0) license, which permits others to distribute, remix, adapt, build upon this work non-commercially, and license their derivative works on different terms, provided the original work is properly cited, appropriate credit is given, any changes made indicated, and the use is non-commercial. See http://creativecommons.org/licenses/by-nc/4.0/.

ORCID iD

Fumito Ito http://orcid.org/0000-0002-6866-671X

\section{REFERENCES}

1 Banchereau J, Palucka AK, Dhodapkar M, et al. Immune and clinical responses in patients with metastatic melanoma to CD34(+) progenitor-derived dendritic cell vaccine. Cancer Res 2001;61:6451-8.

2 Palucka K, Banchereau J. Cancer immunotherapy via dendritic cells. Nat Rev Cancer 2012;12:265-77.

3 Wculek SK, Cueto FJ, Mujal AM, et al. Dendritic cells in cancer immunology and immunotherapy. Nat Rev Immunol 2020;20:7-24.

4 Luo X-L, Dalod M. The quest for faithful in vitro models of human dendritic cells types. Mol Immunol 2020;123:40-59.

5 Saxena M, Balan S, Roudko V, et al. Towards superior dendritic-cell vaccines for cancer therapy. Nat Biomed Eng 2018;2:341-6.

6 Perez CR, De Palma M. Engineering dendritic cell vaccines to improve cancer immunotherapy. Nat Commun 2019;10:5408.

7 Bol KF, Schreibelt G, Rabold K, et al. The clinical application of cancer immunotherapy based on naturally circulating dendritic cells. $\mathrm{J}$ Immunother Cancer 2019;7:109.

8 Osugi Y, Vuckovic S, Hart DNJ. Myeloid blood CD11c(+) dendritic cells and monocyte-derived dendritic cells differ in their ability to stimulate T Iymphocytes. Blood 2002;100:2858-66.

9 Balan S, Ollion V, Colletti N, et al. Human XCR1+ dendritic cells derived in vitro from CD34+ progenitors closely resemble blood dendritic cells, including their adjuvant responsiveness, contrary to monocyte-derived dendritic cells. J Immunol 2014;193:1622-35.

10 Wculek SK, Amores-Iniesta J, Conde-Garrosa R, et al. Effective cancer immunotherapy by natural mouse conventional type-1 dendritic cells bearing dead tumor antigen. J Immunother Cancer 2019;7:100. 
11 Robbins SH, Walzer T, Dembélé D, et al. Novel insights into the relationships between dendritic cell subsets in human and mouse revealed by genome-wide expression profiling. Genome Biol 2008:9:R17.

12 Shortman K, Heath WR. The CD8+ dendritic cell subset. Immunol Rev 2010;234:18-31.

13 Hildner K, Edelson BT, Purtha WE, et al. Batf3 deficiency reveals a critical role for $\mathrm{CD} 8 \alpha+$ dendritic cells in cytotoxic T cell immunity. Science 2008;322:1097-100.

14 Broz ML, Binnewies M, Boldajipour B, et al. Dissecting the tumor myeloid compartment reveals rare activating antigen-presenting cells critical for T cell immunity. Cancer Cell 2014;26:638-52.

15 Roberts EW, Broz ML, Binnewies M, et al. Critical role for CD103(+)/CD141(+) dendritic cells bearing CCR7 for tumor antigen trafficking and priming of T cell immunity in melanoma. Cancer Cell 2016;30:324-36

16 Senju S, Haruta M, Matsumura K, et al. Generation of dendritic cells and macrophages from human induced pluripotent stem cells aiming at cell therapy. Gene Ther 2011;18:874-83.

17 Choi K-D, Vodyanik MA, Slukvin II. Generation of mature human myelomonocytic cells through expansion and differentiation of pluripotent stem cell-derived lin-CD34+CD43+CD45+ progenitors. $J$ Clin Invest 2009;119:2818-29.

18 lizuka-Koga M, Asashima $\mathrm{H}$, Ando $\mathrm{M}$, et al. Functional analysis of dendritic cells generated from T-iPSCs from CD4+ T cell clones of Sjögren's syndrome. Stem Cell Reports 2017;8:1155-63.

19 Sachamitr P, Leishman AJ, Davies TJ, et al. Directed differentiation of human induced pluripotent stem cells into dendritic cells displaying tolerogenic properties and resembling the $\mathrm{CD} 141^{+}$subset. Front Immunol 2017;8:8.

20 Balan S, Arnold-Schrauf C, Abbas A, et al. Large-scale human dendritic cell differentiation revealing Notch-dependent lineage bifurcation and heterogeneity. Cell Rep 2018;24:1902-15.

21 Kirkling ME, Cytlak U, Lau CM, et al. Notch signaling facilitates In vitro generation of cross-presenting classical dendritic cells. Cell Rep 2018;23:3658-72.

22 Saito H, Okita K, Fusaki N, et al. Reprogramming of melanoma tumor-infiltrating lymphocytes to induced pluripotent stem cells. Stem Cells Int 2016;2016:11

23 Seki T, Yuasa S, Oda M, et al. Generation of induced pluripotent stem cells from human terminally differentiated circulating T cells Cell Stem Cell 2010;7:11-14.

24 Saito H, Iwabuchi K, Fusaki N, et al. Generation of induced pluripotent stem cells from human melanoma tumor-infiltrating lymphocytes. J Vis Exp 2016;117:1. doi:10.3791/54375

25 Nakagawa M, Taniguchi Y, Senda S, et al. A novel efficient feederfree culture system for the derivation of human induced pluripotent stem cells. Sci Rep 2014:4:3594.

26 Okita K, Yamakawa T, Matsumura Y, et al. An efficient nonviral method to generate integration-free human-induced pluripotent stem cells from cord blood and peripheral blood cells. Stem Cells 2013;31:458-66.

27 Yamauchi T, Hoki T, Oba T, et al. T-cell CX3CR1 expression as a dynamic blood-based biomarker of response to immune checkpoint inhibitors. Nat Commun 2021;12:1402.

28 Butler A, Hoffman P, Smibert P, et al. Integrating single-cell transcriptomic data across different conditions, technologies, and species. Nat Biotechnol 2018;36:411-20.

29 Wolock SL, Lopez R, Klein AM. Scrublet: computational identification of cell doublets in single-cell transcriptomic data. Cell Syst 2019;8:281-91.

30 Tirosh I, Izar B, Prakadan SM, et al. Dissecting the multicellular ecosystem of metastatic melanoma by single-cell RNA-seq. Science 2016;352:189-96.

31 Aran D, Looney AP, Liu L, et al. Reference-based analysis of lung single-cell sequencing reveals a transitional profibrotic macrophage. Nat Immunol 2019;20:163-72.

32 Novershtern N, Subramanian A, Lawton LN, et al. Densely interconnected transcriptional circuits control cell states in human hematopoiesis. Cell 2011;144:296-309.

33 Brown CC, Gudjonson H, Pritykin Y, et al. Transcriptional basis of mouse and human dendritic cell heterogeneity. Cell 2019;179:846-63.

34 Bourdely P, Anselmi G, Vaivode K, et al. Transcriptional and functional analysis of $\mathrm{CD} 1 \mathrm{C}+$ human dendritic cells identifies a CD163+ subset priming CD8+CD103+ T cells. Immunity 2020;53:335-52.

35 Dutertre C-A, Becht E, Irac SE, et al. Single-cell analysis of human mononuclear phagocytes reveals subset-defining markers and identifies circulating inflammatory dendritic cells. Immunity 2019:51:573-89.

36 Villani A-C, Satija R, Reynolds G, et al. Single-cell RNA-seq reveals new types of human blood dendritic cells, monocytes, and progenitors. Science 2017;356:1. doi:10.1126/science.aah4573

37 Aibar S, González-Blas CB, Moerman T, et al. SCENIC: singlecell regulatory network inference and clustering. Nat Methods 2017;14:1083-6.

38 Liberzon A, Subramanian A, Pinchback R. MSigDB 3.0. Bioinformatics 2011;27:1739-40.

39 Patro R, Duggal G, Love Ml, et al. Salmon provides fast and bias-aware quantification of transcript expression. Nat Methods 2017;14:417-9.

40 Love MI, Huber W, Anders S. Moderated estimation of fold change and dispersion for RNA-seq data with DESeq2. Genome Biol 2014; $15: 550$

41 Saito H, Okita K, Chang AE, et al. Adoptive transfer of CD8+ T cells generated from induced pluripotent stem cells triggers regressions of large tumors along with immunological memory. Cancer Res 2016;76:3473-83.

42 Lee J, Breton G, Oliveira TYK, et al. Restricted dendritic cell and monocyte progenitors in human cord blood and bone marrow. J Exp Med 2015;212:385-99.

43 Breton G, Lee J, Zhou YJ, et al. Circulating precursors of human CD1c+ and CD141+ dendritic cells. J Exp Med 2015;212:401-13.

44 See P, Dutertre C-A, Chen J, et al. Mapping the human DC lineage through the integration of high-dimensional techniques. Science 2017;356 doi:10.1126/science.aag3009

45 Cytlak U, Resteu A, Pagan S, et al. Differential IRF8 transcription factor requirement defines two pathways of dendritic cell development in humans. Immunity 2020;53:353-70.

46 Villar J, Segura E. Decoding the heterogeneity of human dendritic cell subsets. Trends Immunol 2020;41:1062-71.

47 De Smedt M, Taghon T, Van de Walle I, et al. Notch signaling induces cytoplasmic CD3 epsilon expression in human differentiating NK cells. Blood 2007;110:2696-703.

48 Yin X, Yu H, Jin X, et al. Human blood CD1c+ dendritic cells encompass CD5high and CD5low subsets that differ significantly in phenotype, gene expression, and functions. J Immunol 2017:198:1553-64.

49 Hao Y, Hao S, Andersen-Nissen E, et al. Integrated analysis of multimodal single-cell data. Cell 2021:184:3573-87.

50 Satoh T, Toledo MAS, Boehnke J, et al. Human DC3 antigen presenting dendritic cells from induced pluripotent stem cells. Front Cell Dev Biol 2021;9:667304.

51 Sontag S, Förster M, Qin J, et al. Modelling IRF8 deficient human hematopoiesis and dendritic cell development with engineered iPS cells. Stem Cells 2017;35:898-908.

52 Jongbloed SL, Kassianos AJ, McDonald KJ, et al. Human CD141+ (BDCA-3)+ dendritic cells (DCs) represent a unique myeloid DC subset that cross-presents necrotic cell antigens. J Exp Med 2010;207:1247-60.

53 Crozat K, Guiton R, Contreras V, et al. The XC chemokine receptor 1 is a conserved selective marker of mammalian cells homologous to mouse CD8 $\alpha+$ dendritic cells. J Exp Med 2010;207:1283-92.

54 Bachem A, Güttler S, Hartung E, et al. Superior antigen crosspresentation and XCR1 expression define human CD11C+CD141+ cells as homologues of mouse CD8+ dendritic cells. J Exp Med 2010;207:1273-81.

55 Oba T, Long MD, Keler T, et al. Overcoming primary and acquired resistance to anti-PD-L1 therapy by induction and activation of tumor-residing cDC1s. Nat Commun 2020;11:5415.

56 Oba T, Makino K, Kajihara R, et al. In situ delivery of iPSC-derived dendritic cells with local radiotherapy generates systemic antitumor immunity and potentiates PD-L1 blockade in preclinical poorly immunogenic tumor models. J Immunother Cancer 2021;9:e002432.

57 Hammerich L, Marron TU, Upadhyay R, et al. Systemic clinical tumor regressions and potentiation of PD1 blockade with in situ vaccination. Nat Med 2019;25:814-24.

58 Hegde S, Krisnawan VE, Herzog BH, et al. Dendritic cell paucity leads to dysfunctional immune surveillance in pancreatic cancer. Cancer Cell 2020;37:289-307.

59 Patel A, Oba T, Kajihara R, et al. Multimodal intralesional therapy for reshaping the myeloid compartment of tumors resistant to antiPD-L1 therapy via IRF8 expression. J Immunol 2021;207:1298-309.

60 Barnawi R, Al-Khaldi S, Majed Sleiman G, et al. Fascin is critical for the maintenance of breast cancer stem cell pool predominantly via the activation of the Notch self-renewal pathway. Stem Cells 2016;34:2799-813. 